\title{
The Impact of Hidden Liquidity in Limit Order Books*
}

\author{
Stefan Frey ${ }^{\dagger} \quad$ Patrik Sandås ${ }^{\ddagger}$
}

May 30, 2008

\begin{abstract}
We report evidence that the presence of hidden liquidity is associated with greater liquidity in the order books, greater trading volume, and smaller price impact. Limit and market order submission behavior changes when hidden liquidity is present consistent with at least some traders being able to detect hidden liquidity. We estimate a model of liquidity provision that allows us to measure variations in the marginal and total payoffs from liquidity provision in states with and without hidden liquidity. Our estimates of the expected surplus to providers of visible and hidden liquidity are positive and typically of the order of one-half to one basis points per trade. The positive liquidity provider surpluses combined with the increased trading volume when hidden liquidity is present are both consistent with liquidity externalities.

Keywords: Hidden Liquidity; Iceberg Orders; Hidden Orders; Reserve Orders; Limit Order Markets; Limit Order Books; Transparency;
\end{abstract}

JEL Codes: G10, G14

\section{Introduction}

Transparency is, in general, a desirable quality in markets, but there is still no agreement on how much transparency is optimal. In their survey of limit order markets, Parlour and Seppi (2008) describe the transparency choice in limit order markets as a continuum from a closed book, with no

\footnotetext{
${ }^{*}$ We thank the German Stock Exchange for providing access to the Xetra order book data and Uwe Schweickert for his help with the order book reconstruction. Frey gratefully acknowledges financial support from the Deutsche Forschungsgemeinschaft (DFG), and Sandås gratefully acknowledges financial support from the McIntire School of Commerce.

${ }^{\dagger}$ University of Tübingen, e-mail: stefan.frey@uni-tuebingen.de

${ }^{\ddagger}$ University of Virginia and CEPR, e-mail: patriks@virginia.edu
} 
order disclosure, to an open book, with full real-time order disclosure. In practice, most limit order markets use a market design that lies between the two extremes and allows traders to submit hidden liquidity. A prerequisite for assessing the optimality of this market design is a better understanding of how the behavior of market participants change when hidden liquidity is present. We provide that by examining the interaction between visible and hidden liquidity and overall liquidity and trading activity.

The argument for hidden liquidity rests on the idea that some degree of opacity may be needed to attract orders from large traders to the order book. Large traders may be reluctant to submit orders to a fully transparent order book for fear of revealing their trading intentions. Without the option to submit hidden liquidity, the large traders' orders may migrate to off-exchange venues or after-hours trading, or be broken up over time to minimize price impact. With the option to submit hidden liquidity, the argument goes, large traders are more likely to submit their orders to the order book leading to a deeper order book which leads to more trading and more of the gains from trade being realized. But a critical step in the argument, which has not been addressed in the literature, is how any hidden liquidity interacts with the behavior of liquidity demanders and other liquidity providers. The benefits may be small if hidden liquidity from large traders simply displaces liquidity from other liquidity providers. Alternatively, the impact could be negative if uncertainty about the amount of hidden liquidity or the motives of the large traders cause other liquidity providers to back away and submit fewer or smaller limit orders. The same uncertainty may cause liquidity demanders to be more cautious and submit fewer or smaller market orders. We report evidence on how liquidity demanders and other liquidity providers respond to hidden liquidity that lends some new support to the argument for hidden liquidity.

Exchanges often add the option to submit hidden liquidity by creating a different type of limit order that is known as an iceberg order 1 An iceberg order is a limit order that specifies a price, a total order size, and a visible peak size. The peak size is the maximum number of shares that

\footnotetext{
${ }^{1}$ This type of order is known as a reserve order in some markets. Completely hidden orders can be submitted in some markets and some markets (e.g., BATS Trading) allow both reserve and hidden orders. In fixed-income markets there is a type of reserve order known as a expandable limit order that gives the submitter the option but not the obligation to trade more when the initial size has been executed (see Boni and Leach (2004)) but iceberg orders are also used (see Fleming and Mizrach (2008)).
} 
is displayed to the market at any time. The remainder of the iceberg order is not displayed in the order book. When the first peak size has been fully executed, the visible part is immediately replenished by a size equal to the peak size. At a given price level in the order book all displayed order depth has time priority relative to any hidden depth, irrespective of the order entry times. Because of the replenishment rule, which adds a new peak size immediately after the current visible peak size is executed, an iceberg order is likely to be detected, after its first peak executes, by acute observers of the order book. A sequence of events that includes a trade followed by a new order at the same price with a minimal delay is a signal of an iceberg order. Therefore, one might argue that what large traders are able to or choose to hide by using iceberg orders is not so much their intentions to buy or sell the stock but the size of their desired trades.

We measure the impact that iceberg orders have on the order books and the price dynamics using a sample from German Stock Exchange's Xetra platform that includes iceberg and limit orders. On average, order books with one or more iceberg orders at the best quotes have greater visible depth and narrower inside spreads. The price impact of market orders, the market order size, the conditional probability of a buy versus a sell market order, and the expected duration to the next market order also change when iceberg orders are present. For example, when there is an iceberg order at the best ask quote we find a significant reduction in the price impact of buy market orders, a significant increase in the size of buy market orders, a significant increase in the conditional probability of a buy market order, and a shorter expected time until the next buy market order. The net effect is that iceberg orders are associated with increased liquidity supply and demand consistent with a positive liquidity externality.

We estimate a state-dependent model of liquidity provision in a limit order book that takes into account how the priority rules affect the payoffs to visible and hidden liquidity. We extend the empirical approach of Sandås (2001) to allow liquidity provision to depend on the existence of iceberg orders at either the best bid or ask quotes 2 The iceberg state variable also permit differences in the market order flow and the price impact of market orders in line with the empirical regularities

\footnotetext{
${ }^{2}$ Frey and Grammig (2006) implement the tests of Sandås (2001) on the Xetra sample that we use and find qualitatively similar results. The zero-profit conditions are rejected when applied directly to the total and visible order book depths. Neither study considers state-dependence or accounts for the impact of the priority rules with visible and hidden liquidity.
} 
discussed above. We focus on the case in which traders can perfectly detect the presence of iceberg orders but do not observe their total size. We verify that our findings are robust to uncertainty about iceberg orders by implementing our model using the predictions generated by an iceberg detection algorithm instead of the actual knowledge of the icebergs.

Our empirical results show that the expected payoffs of different liquidity provision strategies change with iceberg orders. For example, payoffs to marginal limit orders at the best quotes are negative in order books with no iceberg orders on the same side but positive with iceberg orders on the same side. The first finding is consistent with existing evidence but the second finding, which is new, demonstrates the iceberg orders' indirect impact on the payoffs to liquidity provision. The positive impact of the iceberg order on the payoffs of limit orders is driven by the smaller price impact of market orders that hit the book. The systematic variations in the expected payoffs suggest that liquidity providers do not completely equalize the marginal payoffs across the different order book levels and states.

Based on the model parameter estimates we calculate the surplus that accrues to liquidity providers. Our surplus measure is the expected gain to all limit orders taking into account the probability of the order being executed and the associated price impact. Our surplus estimates are, in general, positive and of the order of one-half to one basis points per trade. Given the increased trading volume when hidden liquidity is present and the positive surplus accruing to liquidity providers it suggests that periods with hidden liquidity are associated with liquidity externalities.

There is a large literature on market transparency which is often classified along the pre-trade versus post-trade dimensions 3 Hidden liquidity is an example of a pre-trade transparency issue but there are several others. The complexity of the issue of pre-trade transparency arises because the nature of the trade-offs involved change with the trading mechanism, the type of information that is disclosed, and the participants to whom information is disclosed 4 A number of studies focus

\footnotetext{
${ }^{3}$ See the sections on market transparency in O'Hara (1995), Madhavan (2000), and Biais, Glosten, and Spatt (2005) for in-depth discussions.

${ }^{4}$ Biais (1993), Madhavan (1995), Madhavan (1996), Pagano and Röell (1996), Bloomfield and O'Hara (2000), Baruch (2005), Moinas (2006), Foucault, Moinas, and Theissen (2007) among others develop theoretical models of transparency and Flood, Huisman, Koedijk, and Mahieu (1999) and Bloomfield and O'Hara (1999) carry out experimental studies of trading in different transparency regimes. A number of empirical studies including Anand and Weaver (2004), Boehmer, Saar, and Yu (2005), Foucault, Moinas, and Theissen (2007), Hendershott and Jones (2005), Madhavan, Porter, and Weaver (2005) focus on the impact of changes in market transparency.
} 
specifically on hidden orders or iceberg orders. Pardo and Pascual (2006) and Tuttle (2006) find evidence consistent with iceberg orders being perceived as uninformed orders. A number of studies have focused on different aspects of the decision problem faced by a submitter of an iceberg order. Esser and Mönch (2007) focus on the trade-off between price and peak size of the iceberg order. Bessembinder, Panayides, and Venkataraman (2008) and D'Hondt and De Winne (2007) study how the decision to not display the full order interacts with other dimensions of the trader's order choice problem. Harris (1996) studies how variations in the minimum tick size influence the willingness to display larger order quantities. Aitken, Berkman, and Mak (2001) examine variation in the use of hidden orders around a change in the threshold size for such orders. We add to this literature by focusing on how the presence of iceberg orders influence the payoffs and the strategies of other liquidity providers and how liquidity demanders respond to the presence of hidden liquidity.

\section{Our Sample}

Our sample includes all order entries, trades, and cancellations in the thirty stocks in the DAX-30 German blue chip index for the period January 2nd to March 31st, 2004. Our sample is from the Frankfurt Stock Exchange's electronic trading platform Xetra and provides detailed information that enables us to reconstruct complete histories for individual orders and for the visible and hidden portions of the limit order books. Table A1, in the appendix, reports descriptive statistics for the sample stocks. In 2004, trading on Xetra accounted for approximately $75 \%$ of all domestic equity trading. Equity trading off the Xetra platform was split between over-the-counter trading, which accounted for approximately 19\%, and, floor trading and trading on regional exchanges, which together accounted for approximately $6 \% 5$

On Xetra, traders can, in addition to market and limit orders, submit iceberg orders 6 An

\footnotetext{
${ }^{5}$ The statistics for overall domestic equity trading in Germany are based on the statistics reported by the Federation of European Securities Exchanges (http://fese.eu) and by the Deutsche Börse Group (2005 Factbook).

${ }^{6}$ Iceberg orders were added to the Xetra system in 2000, three years after its introduction. Other examples of markets that have introduced iceberg orders or converted other the types of hidden liquidity to iceberg orders after introducing an electronic limit order book include the London Stock Exchange, which introduced iceberg orders in 2003, and the Toronto Stock Exchange, which reintroduced iceberg orders in 2002 after a six-year period without iceberg orders. The Australian Stock Exchange recently replaced its undisclosed order type with an iceberg order. Recently, the New York Stock Exchange proposed to add reserve orders that are similar to iceberg orders. Securities Industry News, March 31st, 2008, "NYSE Will Offer Electronic Reserve Orders."
} 
iceberg order specifies a price, a total size, and a peak size. The peak size is the maximum visible volume of the order. When a trader submits an iceberg order, the first peak size is visible in the order book. At that time, the hidden volume of the order is equal to the order's total size minus its peak size. When the first peak size has been fully executed, the visible part is automatically replenished by a number of shares equal to the peak size, and the hidden part is reduced by the corresponding number of shares. The replenishment of the visible part continues automatically until the hidden volume is depleted or the trader cancels the iceberg order.

In the order book, an order is given priority according to price, display condition, and time. A sell order at a lower price has priority relative to any sell orders at higher prices, irrespective of the order's time of submission or display condition. At the same price level, a displayed order has priority relative to any hidden orders regardless of the order's time of submission. Among displayed orders, an order submitted earlier has priority relative to any orders submitted later. When an iceberg order's visible part is replenished and the next peak size converts from hidden to displayed status the newly visible peak size also receives a new time stamp which determines its time priority.

We reconstruct the sequence of order books from the event histories in the sample. The order records include a flag for an iceberg order which we use to construct complete histories for all limit and iceberg orders. From these histories we reconstruct snapshots of the visible and hidden order books before each transaction. In addition, we construct individual order histories that we use to examine the placement, execution, cancellation, and duration of limit and iceberg orders. We restrict our sample to orders submitted during the continuous trading period. Continuous trading on Xetra starts after an opening auction, ends with a closing auction, and stops for a few minutes, in the middle of the day, for an auction. The reconstruction takes into account the effects that any order submissions, transactions, or cancellations in the auctions have on the state of the order book during continuous trading.

Iceberg orders are designed to solve a problem of transacting a large number of shares so we expect the orders to be larger than limit orders, but they may also differ along other dimensions. Tables 1 and 2 report descriptive statistics that show some of the differences between iceberg and limit orders. The iceberg orders' share of all submitted and executed shares are reported in 
Table 1. We exclude all market orders and any marketable limit or iceberg orders in calculating the percentages. Across all stocks, iceberg orders represent $9 \%$ of all shares submitted, but they represent $16 \%$ of all shares executed implying a higher execution rate for iceberg orders than for limit orders. The higher execution rate cannot be explained by differences in order size since both measures are expressed in numbers of shares rather than in number of orders. They may, however, be driven by differences in the price (order placement) and duration of iceberg orders.

The rightmost two columns of Table 1 report the median distance between the same-side best quotes and the iceberg and limit order prices. There is not a clear pattern in the median distances. While the iceberg orders, on average, are placed closer to the best quotes for large and medium stocks the evidence is mixed for the smaller stocks. Overall, the cross-sectional average of the median distances is 3.6 basis points for iceberg orders and 3.9 basis points for limit orders. An average difference of 0.3 basis points is small in comparison to the average half-spread of 3.6 basis points (Table A1). The relatively small difference and the mixed ordering across sub-samples suggest that a difference in the typical order placement is not likely to be the main driver of differences in execution rates.

The middle four columns (columns four through seven) of Table 1 report statistics on the order sizes. The last entries of the fourth and fifth columns report that, on average, the limit order size is a 1,000 shares whereas the peak size of an iceberg order is 2,600 shares implying that the visible part of an iceberg order is between two and three times the size of a typical limit order. The sixth column, labeled 'Total Size/Peak Size,' reports the average of the ratio of the iceberg order's total size to its peak size. The average ratios are with one exception between 5 and 10 which reflects clustering at even multiples such as five or ten times the peak size. The column labeled 'Executed Shares/Peak Size' reports the ratio of executed shares to peak size for all iceberg orders whose first peak size was executed. The average ratio of 4.6 implies that, on average, iceberg orders are replenished almost four times conditional on the first peak size being executed and therefore almost $80 \%$ of the executed iceberg shares originate from initially hidden volume.

Panel A of Table 2 reports the percentage of limit and iceberg orders that are either partially or fully executed. Forty-seven percent of all iceberg orders receive at least a partial execution whereas 
the corresponding figure for limit orders is only fourteen percent. The last column of Panel A reports the ratio of the median duration for iceberg orders to the median duration of limit orders. The ratio of median durations of 7.1 shows that iceberg orders spend a substantially longer time in the order book. Holding other things equal, one would expect that a longer time spent in the order book would lead to a greater chance of order execution. Another implication of a longer duration is that the fraction of limit order books that contain iceberg orders is greater than the fraction of shares submitted that are iceberg orders.

We create two sub-samples of iceberg orders and matched limit orders to determine if the execution of iceberg and limit orders differs after the first peak of an iceberg order is executed. Panel B of Table 2 reports, for two sub-samples, the execution rates and the ratio of median timeto-fill for iceberg and limit orders. The samples are selected to isolate possible differences in the information that is available to the market as a function of how long the iceberg order has been in the order book.

The sub-sample labeled 'First Peak' consists of iceberg orders that at the time of the observation (i) have zero shares executed, (ii) have a peak size that differs by $10 \%$ or less from the modal peak size, and (iii) have an order price relative to the same-side best quote that falls between the 30th and 70th percentile for all iceberg orders. The matching limit order sample includes all limit orders with order sizes and quantities that fall within the price and size cut-offs for the iceberg orders. The sub-sample labeled 'Second Peak' includes only iceberg orders whose first peak size, at the time of the observation, has been executed. The execution of the first peak implies that, at the time of the execution, the iceberg order was at the front of the order queue. Accordingly, in the matching limit order sample we keep only the limit orders that undercut the best quote and therefore also are at the front of the order queue.

The execution frequencies for the First Peak sample are comparable with an average of $86 \%$ for limits and $90 \%$ for iceberg orders. The average ratio of the median time-to-fill is 1.0 implying that iceberg orders that are less likely to be detected are similar to otherwise similar limit orders along these two dimensions. The execution frequencies for the Second Peak sample are also comparable for the matched iceberg and limit order samples although the relative ordering is reversed relative 
to the First Peak. The time-to-fill, however, is shorter for iceberg orders with an average ratio of 0.7. Out of the thirty ratios, 23 are below 1 and only one ratio is above one (TUI). Iceberg orders that are more likely to have been detected appear to attract market orders generating executions more rapidly than otherwise comparable limit orders. Overall, the higher execution frequencies of iceberg orders may reflect both the longer order durations and a tendency for iceberg orders to attract market order flow.

\section{Order Books, Price Dynamics and Iceberg Orders}

The hidden depth, larger size, and longer duration of iceberg orders may lead them to have a significant influence on both the order books and the price dynamics around trades. In this section, we examine more closely the interaction between iceberg orders, the limit order books, the price impact of market orders, and the market order flow. For tractability we make some simplifying assumptions. We condition on whether or not an iceberg order is present at the best bid or ask quotes, but we do not condition on the size of hidden depth. We focus on changes in the visible depth in the order books with iceberg orders since, by definition, such order books have additional hidden depth (See, Table 1). We restrict the focus to the two best bid and ask levels and iceberg orders at the best levels.

\subsection{The Limit Order Books and Iceberg Orders}

Table 3 reports the average spreads and visible depths observed before transactions, conditional on whether or not the order book contains iceberg orders. The order book snapshots are created $1 / 100$ th of a second before every transaction. Panel A reports that between 18 and $26 \%$ of all order book snapshots have at least one iceberg order at either the best bid or ask quotes. For each stock and iceberg scenario we compute an average spread and depth and we then compute and report the cross-sectional means of the individual averages for the three categories; large, medium, and small stocks. We also report the difference in the cross-sectional means and the number of stocks that have a difference that is significantly different from zero and of the same sign as the overall difference. 
Panel B shows that the mean bid-ask spread for large stocks is 4.9 basis points for books without iceberg orders and 4.1 basis points for books with at least one iceberg order on either the bid or the ask side. Across all three groups, the mean spreads without iceberg orders are 0.7 to 1.2 basis points wider than the spreads with one or more iceberg orders in the book. For all stocks, we reject the null hypothesis of the difference in spreads being zero. The spread between the best and second best price levels in the order book is narrower when there is an iceberg order at the opposite side of the book, but it is wider when there is an iceberg order at the same side of the book. The spread is 0.2 to 0.4 basis points narrower with the iceberg order at the opposite side and we reject the null of no difference for 27 stocks. The difference is greater in magnitude when the iceberg order is at the same side; the difference ranges from -0.6 to -1.4 basis points. The most common iceberg scenario is an iceberg order at one side of the order book. In that scenario, the net effect-adding the change for the inside spread and the spreads between the best and second best quotes - is a narrower spread for order books with iceberg orders.

Panel $\mathrm{C}$ shows that the visible depth at the best quote is greater when an iceberg order is present at the best quote at either the opposite or the same side of the book with a greater increase when the iceberg order is at the same side. The null hypothesis of no difference is rejected for 29 stocks in the same side case and for 23 stocks for the opposite side case. The magnitude of the increase in the same side case corresponds to approximately an average-sized limit order and thus is less than the peak size of a typical iceberg order. That means that traders submit fewer or smaller limit orders when an iceberg order is present, but that the drop is small enough to increase the net depth. The visible depth at the second level does not change much between books with and without iceberg orders, with the exception of the medium stocks and iceberg orders at the opposite side.

\subsection{The Price Impact and Iceberg Orders}

We estimate price impact regressions that allow the price impact of a market order to depend on whether or not there is one or more iceberg orders in the limit order book. Let $\Delta m q_{+\tau}$ denote the change in the mid-quote, $m q$, from time $t$ to $t+\tau$ measured in basis points. We denote the size of 
the market order by $m ; m>0$. The market order size is normalized for each stock so that $m$ is measured in units of the average market order size. Let $d$ denote the sign of the market order at time $t, d=+1$ for a buy market order, and $d=-1$ for a sell market order.

Let $I^{\text {bid }}$ be an indicator that takes on a value of one, if there is at least one iceberg order at the best bid level in the limit order book at time $t$. Let $I^{a s k}$ be the corresponding indicator order for the best ask level. We focus on a symmetric case in which the magnitude of the price impact of a buy market order when an iceberg order is at the best ask is the same as that of a sell market order when an iceberg order is at the best bid, holding other things equal. We use the indicators $I^{h}$ and $I^{n h}$ to differentiate the cases in which an iceberg order is at the side that the market order hits or at the opposite side. If a buy market order arrives $(d=+1)$, we set $I^{h}=I^{a s k}$ and $I^{n h}=I^{b i d}$, and, if a sell market order arrives $(d=-1)$, we set $I^{h}=I^{b i d}$ and $I^{n h}=I^{a s k}$.

We estimate the regressions for the following three time horizons: 10 minutes, 30 trades, and next trade. We include the next trade case primarily as a benchmark because such a short horizon is likely to be influence by mechanical effects due to the replenishment of iceberg orders. The longer time horizons obviously add more noise but they are also less likely to pick up such mechanical effects. If the time horizon goes beyond the closing time we use the closing price as the revised mid-quote.

We estimate the following regression for each stock:

$$
\Delta m q_{+\tau}=c+\left(a_{0}+a_{1} m\right) d+\left(a_{0}^{h}+a_{1}^{h} m\right) d I^{h}+\left(a_{0}^{n h}+a_{1}^{n h} m\right) d I^{n h}+\epsilon_{+\tau} .
$$

The baseline price impact without iceberg orders is determined by the parameters $a_{0}$ and $a_{1}$. The parameters $a_{0}^{h}$ and $a_{1}^{h}$ capture any change in the price impact function for the case in which the market order hits the order book side with an iceberg order. The parameters $a_{0}^{n h}$ and $a_{1}^{n h}$ capture any change for the case in which the order book side that is not hit has an iceberg order.

Table 4 reports the average parameter estimates and standard errors for the large, medium, and small sub-samples for the three time horizons. The magnitude of the estimates of $a_{0}$ and $a_{1}$ increase from large to small stocks and from next trade to either the 10 minute or the 30 trade horizons. The average estimated baseline price impact for a unit market order and the 30 trade 
horizon — adding $a_{0}$ and $a_{1}$ —is $2.3,3.3$, and 4.2 basis points for the large, medium, and small stocks. The corresponding average impacts are almost identical for the 10 minute horizon. The change in the fixed and variable price impact $\left(a_{0}^{h}\right.$ and $\left.a_{1}^{h}\right)$ when a market order hits the order book side with an iceberg order $\left(I^{h}\right)$ are both negative with estimates that are of a larger magnitude for the two longer horizons. For example, for the large stocks the average expected net price impact of a unit market order for the 30 trade horizon drops by approximately $80 \%$ from 2.2 to 0.3 basis points. The estimates for the parameter for the fixed component when an iceberg is present on the order

book side not hit by the market order $\left(a_{0}^{n h}\right)$ are, on average, positive and range from 1.5 to 4.3 basis points for the 30 trade horizon. The variable component $\left(a_{1}^{n h}\right)$ is on average negative but typically not significantly different from zero. The net effect when an iceberg order is present at side of the book not hit by the market order is therefore to increase the expected price impact by between 1.5 to 4.3 basis points.

The bottom panel of Table 4 report results for an extended version of the price impact regression that allows for asymmetric effects for buy and sell orders. The number of stocks for which the joint hypothesis of asymmetric fixed effects (different $a_{0}$ coefficients) and variable effects (different $a_{1}$ coefficients) are rejected at the $1 \%$ level are reported. The evidence in favor of an asymmetric price impact function is weak. The parameter estimates for the asymmetric effect, which are not reported, are in general small in magnitude relative to the symmetric case even in the cases that reject the null.

\subsection{The Market Order Flow and Iceberg Orders}

In order to determine to what extent the characteristics of market orders change with icebergs in the books we examine the size, direction, and duration for market orders for order books with and without iceberg orders. To determine whether the market order size changes when iceberg orders are present we regress the market order size on iceberg indicators. We report results for specifications with symmetric buy and sell side effects. We have also estimated asymmetric specifications but the asymmetries are small relative to the parameters in the symmetric specification. Panel A of Table 5 reports the average parameter estimates and standard errors. The average estimates of 
$c$ range from 0.92 and 0.95 which means that, on average, market orders are $5-8 \%$ smaller for observations without iceberg orders. The estimates for $I^{h}$ are positive and range from 0.37 to 0.50 implying that when there is an iceberg order at the side hit by the market order we observe $37 \%$ to $50 \%$ larger market order quantities. The corresponding average estimates of the parameter on $I^{\text {nh }}$ range from 0.12 for the large to 0.21 for the small stocks implying that even when the iceberg order is at the side not hit by the market order we observe an increase in market order quantities of 12 to $21 \%$. The larger market order sizes in either direction may partly explain the greater price impact for market orders that hit the non-iceberg side of the order book reported in Table 4 .

In order to determine whether the presence of iceberg orders also affects the direction of the market order flow we estimate a logit model of the probability of a buy market order. Panel B reports the average marginal effects, based on the logit model estimates, with standard errors in parentheses. The marginal effects are positive for $I^{h}$ and negative for $I^{n h}$. The positive marginal effect for $I^{h}$ ranges from 0.14 to 0.16 and implies that, if the baseline probability is one-half, the probability of observing a buy market when there is an iceberg at the best ask is between 0.64 and 0.66. The negative marginal effect for $I^{n h}$ ranges from -0.13 to -0.15 implying that, with the same baseline assumption, the probability of a buy market is between 0.35 and 0.37 when there is an iceberg at the best bid.

Panel C reports the cross-sectional means of the parameter estimates for a log-ACD (Autoregressive Conditional Duration) model for the expected duration between market orders 7 Following the literature, we remove time of day effects using a spline function for thirty minute intervals. Our specification differs from the baseline only in terms of the choice of additional explanatory variables, which in our case are $I^{h}$ and $I^{n h}$. The parameter estimates for $\omega, \alpha$, and $\beta$, while not our primary focus, are comparable to the ones reported in Bauwens and Giot (2000) for NYSE stocks. The average estimates for $I^{h}$ are negative ranging from -0.05 to -0.09 implying a 5 to $9 \%$ decrease in the normalized expected duration when there is an iceberg order at the side of the book hit by the market order. The corresponding average estimates are positive for $I^{n h}$, but smaller in magnitude, ranging from 0.02 to 0.04 implying a 2 to $4 \%$ increase in the expected normalized duration when

\footnotetext{
${ }^{7}$ Bauwens and Giot (2000) present the log-ACD model as an extension of the ACD model introduced by Engle and Russell (1998) that allows for other explanatory variables to determine the durations without a sign restriction.
} 
there is an iceberg at the side of the order book that is not hit by the market order.

\subsection{Interpretation}

The above results suggest that the presence of iceberg orders is associated with greater visible depth and narrower spreads in the limit order book. Hidden liquidity is not simply displacing visible liquidity in the order books. The presence of iceberg orders is associated with larger market orders that tend to be skewed towards the side with the iceberg order. The price impact for a market order that hits the side with an iceberg order is less than for an otherwise similar market order suggesting that liquidity demanders may adjust their trading strategies to take advantage of the variation in liquidity. Together these effects suggest that the relative payoffs to providing liquidity may vary along several dimensions in order books with iceberg orders. In the next section we present a simple model that allows us to incorporate the price, display, and time priority rules that determine how visible and hidden orders interact in the order book and to determine the net effects on the marginal payoffs to liquidity provision.

\section{Model}

Our model captures several key features that influence the interaction between visible and hidden depth in limit order books. The model incorporates a price, display, and time priority rule and captures the discriminatory nature of limit order executions. The model permits the price impact function and the market order distribution to depend on whether or not there are iceberg orders in the book.

\subsection{Model Structure}

Two types of players submit orders to the limit order book. Liquidity providers submit limit orders to the order book and earn a compensation for their liquidity provision that depends on the value of the stock conditional on their limit orders executing. Large traders may provide hidden liquidity by submitting iceberg orders to the order book. We abstract from the large trader's motivation for trading and the details of her decision problem and focus instead on the response of the liquidity 
providers. From the liquidity providers' perspective the arrival and replenishment of iceberg orders follow some exogenous stochastic process. The expected payoffs to the liquidity providers depend on the probability of a market order, the distribution of market order sizes, the limit order's position in the order book, and the possible size and location of any iceberg orders. All liquidity providers agree on a fundamental value of $X$ for the stock at time $t ; X$ may be interpreted as the liquidity providers' time $t$ expectation of the liquidation value of the stock.

The time $t$ market order is submitted by a trader who may be informed about the future value of the stock. As in Section 3.2 we denote the size of the time $t$ market order by $m$, and the direction of the market order by $d$.

The following three components determine the observed change in the fundamental value between $t$ and $t+\tau$ : a drift term $\mu$, private information revealed by the market order flow, and new public information. The new fundamental value at $t+\tau, X_{+\tau}$, is given by:

$$
X_{+\tau}=X+\mu+\left(\alpha_{0}+\alpha_{1} m\right) d+\epsilon_{+\tau}
$$

in which, $\alpha_{0}$ and $\alpha_{1}$ are parameters that measure the information content of the market order flow, $d$ is plus one for a buy market and minus one for a sell market, and $\epsilon_{+\tau}$ is a mean-zero, independent random variable that reflects the impact of public news that arrives between $t$ and $t+\tau$.

The bid and ask sides of the limit order book at time $t$ are characterized by a series of quotes, $p^{1}, p^{2}, \ldots, p^{K}$, with the index starting from the best quote. The total visible volume offered at the $k$ th best quote is denoted by $q^{k}$. The cumulative visible volume offered at all quotes with equal or higher priority to the $k$ th best quote, $p^{k}$, is denoted by $Q^{k}$, and is determined as, $Q^{k}=\sum_{i \leq k} q^{i}$. Accordingly the hidden volumes are denoted by $\hat{q}^{k}$ and the cumulative hidden volumes by $\hat{Q}^{k}$. In our model we focus primarily on the hidden volume at the best quotes. Since liquidity providers are risk neutral they care about the expected hidden depth, which we assume is the same for the bid and ask side and denote by $\eta=E\left[\hat{q}_{1} \mid \hat{q}_{1}>0\right]$.

We use the indicators $I^{o w n}$ and $I^{o p p}$ to differentiate the cases in which an iceberg order is at the 'own' side and 'opposite' side viewed from the perspective of a given limit order in the order book. Let $I^{\text {own }}$ be an indicator for an iceberg order on the own side, and let $I^{\text {opp }}$ be an indicator 
for an iceberg order on the opposite side. It is convenient to summarize the information about the state of the order book by a vector $h$ that is defined as $h^{\prime}=\left[\begin{array}{ll}1 & I^{\text {own }} I^{\text {opp }}\end{array}\right]$. The baseline case of no iceberg orders at either side leads to $h^{\prime}=\left[\begin{array}{lll}1 & 0 & 0\end{array}\right]$. In the model traders know whether or not the book contains iceberg orders at the best bid and ask quotes, i.e., they observe the value of $h$. Section A1, in the appendix, provide a more detailed discussion on how traders may use market data to detect the presence of iceberg orders.

\subsection{The Order Book Without Iceberg Orders}

Limit orders are executed in a discriminatory fashion. A limit sell order that is executed if a buy market order of size $m$ arrives is also executed by any larger buy market order. Upper- and lowertail expectations of the fundamental value determine the expected revision in the fundamental value conditional on execution (as in Glosten (1994)). Given the symmetry assumption we define a single tail expectation that we denote by $x_{+\tau}(m, d ; \theta)$, which is defined as the expected value of $X$ at $t+\tau$, conditional on a market order at time $t$ of size $m$ or greater. Combining the affine price impact function of Equation (2) with the direction of the market order, $d$, the tail expectation $x_{+\tau}(m, d ; \theta)$ is given by:

$$
\begin{aligned}
x_{+\tau}(m, d ; \theta) & =E\left[X_{+\tau} \mid X, \tilde{m} \geq m, d\right] \\
& =X+\mu+\left(\alpha_{0}+\alpha_{1} E[m \mid \tilde{m} \geq m]\right) d
\end{aligned}
$$

with $\theta$ denoting the vector of parameters including $\mu, \alpha_{0}$, and $\alpha_{1}$, and any parameters for the distribution of $m$. The expected marginal payoff conditional on execution for the marginal order at the best ask quote is denoted by $\delta_{1}^{\text {vis }}$ and is given by

$$
\begin{aligned}
\delta_{1}^{v i s} & =E\left[\left(p^{1}-X_{+\tau}\right) \mid m \geq q^{1}\right] \\
& =p^{1}-x_{+\tau}\left(q^{1}, d=1 ; \theta\right)
\end{aligned}
$$

A similar equation can be derived for the best bid quote. The corresponding expected payoff for a marginal limit at the $k$ th best level is obtained by replacing the single tail expectation, $x_{+\tau}\left(q^{1}, d ; \theta\right)$ 
in Equation (44), by $x_{+\tau}\left(Q^{k}, d ; \theta\right)$. Under perfect competition the $\delta$ 's would be driven to the pershare order processing cost.

\subsection{The Order Book With Iceberg Orders}

With iceberg orders we have four possible states and the price impact function is characterized by the parameter vectors $\alpha_{0}=\left[\alpha_{0}^{\text {vis }} \alpha_{0}^{\text {own }} \alpha_{0}^{o p p}\right]$ and $\alpha_{1}=\left[\alpha_{1}^{\text {vis }} \alpha_{1}^{\text {own }} \alpha_{1}^{o p p}\right]$. The discounts or premia for marginal liquidity provision are also state dependent and we refer to them as the marginal payoffs. The case of no iceberg orders is the baseline case with marginal payoffs, $\delta_{1}^{v i s}$ and $\delta_{2}^{\text {vis }}$. The parameter $\delta_{1}^{\text {own }}$ measures the change in the marginal payoff, relative to the baseline case, when there is an iceberg order at the same side of the book. The parameter $\delta_{1}^{o p p}$ measures the corresponding change in the marginal payoff when the iceberg order is present at the opposite side of the order book. The

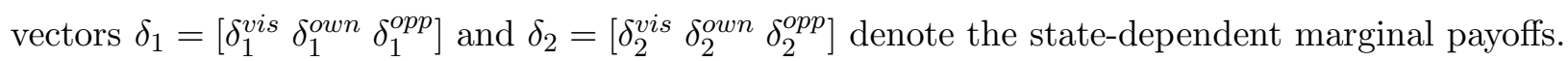

The state-dependent single tail expectations for the best and second-best quotes, $x_{+\tau}\left(q^{1}, d ; \theta\right)$ and $x_{+\tau}\left(Q^{2}, d ; \theta\right)$, take the following form:

$$
\begin{aligned}
x_{+\tau}\left(q^{1}, d ; \theta\right) & =X+\mu+\left(\alpha_{0} h+\left(\alpha_{1} h\right)\left(E\left[m \mid m \geq q^{1}, h\right]\right)\right) d \\
x_{+\tau}\left(Q^{2}, d ; \theta\right) & =X+\mu+\left(\alpha_{0} h+\left(\alpha_{1} h\right)\left(E\left[m \mid m \geq Q^{2}, h\right]+I^{o w n} \eta\right)\right) d .
\end{aligned}
$$

In the first tail expectation the indirect effect of an iceberg order is captured through shifts in the price impact per unit of market order measured by $\alpha_{0} h$ and $\alpha_{1} h$ and by shifts in the distribution of market order quantities measured by $E\left[m \mid m \geq Q^{2}, h\right]$. The same indirect effects apply to the second tail expectation but in addition there is the direct effect of the hidden liquidity captured by the last term $I^{o w n} \eta$.

\subsection{Liquidity Provider Surplus}

The $\delta$ parameters in our model capture the marginal compensation, conditional on execution, for the marginal units at different levels in the order book. The expected surplus to all units take into account the probability of execution and the corresponding tail expectations. Let $\pi^{k}(q)$ denote the expected surplus for the $q$ th unit at quote k. Using Equation (3) we can write the surplus $\pi^{k}(q)$ 
for the ask side as

$$
\pi^{k}(q)=\int_{q}^{\infty}\left[p^{k}-x_{+\tau}(m, d=1 ; \theta)\right] \operatorname{Pr}(d=1 ; h) f(m ; h, \lambda) d m
$$

where $\operatorname{Pr}(d=1 ; h)$ denotes the state dependent probability of a buy order and $f(m ; h, \lambda)$ the probability distribution of the size of market orders with parameter vector $\lambda$. An equivalent formula is used for the bid side.

Denote the aggregate expected surplus for the visible volume at quote $\mathrm{k}$ by $\Pi^{k}$. We obtain it by integrating $\pi^{k}(q)$ for the visible volume at quote $\mathrm{k}$

$$
\Pi^{k}=\int_{Q^{k-1}+\hat{Q}^{k-1}}^{Q^{k}+\hat{Q}^{k-1}} \pi^{k}(q) d q
$$

where we use the definition of $Q^{0}=0$ and $\hat{Q}^{0}=0$.

The aggregate expected surplus for the hidden volume at quote $\mathrm{k}$ is $\hat{\Pi}^{k}$ and calculated by

$$
\hat{\Pi}^{k}=\int_{Q^{k}+\hat{Q}^{k-1}}^{Q^{k}+\hat{Q}^{k}} \pi^{k}(q) d q
$$

The total liquidity provider surplus is obtained by adding across quotes of both sides of the order book. By conditioning on the state or a subset of order book levels we can calculate various components of the total surplus.

\section{Empirical Results}

We start by briefly describing the parametrization of our model and our estimation strategy. We then present the estimates of the model parameters and the marginal payoffs to liquidity provision. Finally, we present our estimates of the expected surplus to liquidity providers. 


\subsection{Market Order Distribution}

For the distribution of the market order size $f(m ; \lambda, h)$ we choose the exponential distribution with state dependent parameter vector $\lambda=\left(\lambda^{\text {vis }}, \lambda^{\text {own }}, \lambda^{\text {opp }}\right)$. The expected mean depends on the state vector $h: E[m ; h]=\lambda h$, where $\lambda^{o w n}$ and $\lambda^{o p p}$ equals the change of the expected mean from the visible to the own and opposite iceberg states.

The state dependent probability of a buy market order $\operatorname{Pr}(d=1 ; h)$ is estimated by the state dependent sample probabilities of buy market orders.

\subsection{Estimation Strategy}

We use the restrictions implied by the model to specify a set of moment conditions that we denote by $g(\cdot)$. The state-dependent marginal payoffs generate a set of moment conditions that involve the lower-tail and upper-tail expectations, the $\delta$ parameters, and the iceberg state vector $h$. Here we use superscripts for the $h$ vector to distinguish between the state viewed from the bid and the ask sides of the order book:

$$
\begin{aligned}
& g^{1}(\theta)=E\left[\left[\begin{array}{l}
x_{+\tau}\left(q^{1}, d=-1 ; \theta\right)-p^{1}-\delta_{1} h^{b i d} \\
x_{+\tau}\left(Q^{2}, d=-1 ; \theta\right)-p^{2}-\delta_{2} h^{b i d}
\end{array}\right] \otimes h^{b i d}\right]=0 . \\
& \quad \text { and } \\
& g^{2}(\theta)=E\left[\left[\begin{array}{l}
p^{1}-x_{+\tau}\left(q^{1}, d=+1 ; \theta\right)-\delta_{1} h^{a s k} \\
p^{2}-x_{+\tau}\left(Q^{2}, d=+1 ; \theta\right)-\delta_{2} h^{a s k}
\end{array}\right] \otimes h^{a s k}\right]=0 .
\end{aligned}
$$

The state-dependent price impact and market order flow imply the following set of moment conditions:

$$
g^{3}(\theta)=E\left[\left[\begin{array}{l}
m-\lambda h^{m k t} \\
X_{+\tau}-X-\mu-\left(\alpha_{0} h^{m k t}\right) d-\left(\alpha_{1} h^{m k t}\right)(d \cdot m) \\
\left(X_{+\tau}-X-\mu-\left(\alpha_{0} h^{m k t}\right) d-\left(\alpha_{1} h^{m k t}\right)(d \cdot m)\right) d \\
\left(X_{+\tau}-X-\mu-\left(\alpha_{0} h^{m k t}\right) d-\left(\alpha_{1} h^{m k t}\right)(d \cdot m)\right)(d \cdot m)
\end{array}\right] \otimes h^{m k t}\right]=0
$$

Finally, the hidden depth, $\eta$, is identified from the following two moment conditions:

$$
g^{4}(\theta)=E\left[\begin{array}{l}
\left(\hat{q}^{a s k}-\eta\right) 1\left(\hat{q}^{a s k}>0\right) \\
\left(\hat{q}^{b i d}-\eta\right) 1\left(\hat{q}^{b i d}>0\right)
\end{array}\right]=0
$$


We replace $X$ and $X_{+\tau}$ by the mid-quotes observed immediately before the transactions at times $t$ and $t+\tau$. We use the 30 trade time horizon. Using the 10 minute horizon yields similar results. We stack the moment conditions, $g^{1}(\cdot), \ldots, g^{4}(\cdot)$ and estimate the model parameters using GMM in two stages. In the second-stage estimation we use a Newey-West type weighting matrix with a Bartlett kernel with 10 lags. Table 6 summarizes the parameters and the moment conditions of our model.

\subsection{Model Parameter Estimates}

We estimate the model separately for each stock and report average parameter estimates and standard errors for the large, medium, and small categories in Table 7 . Panel A of Table 7 reports the estimates for the price impact function. Overall, the parameter estimates for the price impact function are very close to the estimates for the price impact regression reported in Table 4 (for the 30 trade horizon). The price impact of market orders changes with the presence of iceberg orders. When an iceberg order is present on the side that is hit by the market order (own side), the net fixed component of the price impact is approximately zero and the variable component impact

drops to approximately half of its baseline value. When the iceberg order is on the side opposite to the side hit by the market order (opposite), the fixed component increase by between 65 and $100 \%$ whereas the variable component stays essentially unchanged.

Panel B reports the estimates for the marginal payoffs to liquidity provision at the best and second best levels. On average, the $\delta_{1}^{\text {vis }}$ estimates are negative and range from 0.8 to 1.6 basis points. One interpretation of the negative estimates is that, on average, traders who determine the marginal prices at the best bid or ask level have some intrinsic reason for trading. Accepting a negative payoff of this magnitude is rational if the alternative is to pay one-half of the bid-ask spread, which range from approximately 2.4 basis points for the large category to 4.3 basis points for the small category. The estimates of $\delta_{2}^{v i s}$ are positive and range from 1.2 to 2.4 basis points implying that liquidity providers at the second best level expect to have a positive net payoff after accounting for the price impact.

The average estimates of the own side marginal payoffs, $\delta_{1}^{o w n}$ and $\delta_{2}^{\text {own }}$, are positive across all 
three categories albeit that only the $\delta_{1}^{\text {own }}$ estimates are significantly different from zero for most stocks. The positive marginal payoff for liquidity providers at the best level is consistent with the reasoning behind some form of order matching or front running strategy (Harris (1996)). Yet, the fact that the marginal payoff is significant and positive would suggest these opportunities are not fully exploited.

The average estimates of the opposite side marginal payoffs, $\delta_{1}^{o p p}$ and $\delta_{2}^{o p p}$, are all negative and, in general, significantly different from zero. The negative payoffs for marginal limit orders in order book on the side opposite an iceberg order may reflect the 'buffer effect' of the iceberg order which limits any favorable price movements for these limit orders.

Panel $\mathrm{C}$ of Table 7 reports the estimates for the mean market order size and hidden depth. The estimates for the mean market order size - the $\lambda$ 's - are close to the regression results reported above in Table 5. The market order sizes are larger when iceberg orders are present with a stronger effect when the iceberg order is on the side hit by the market order. The mean hidden depth ranges from 9 to 14 times the normal market order size.

\subsection{Marginal Payoffs for Liquidity Provision}

We compute the net marginal payoffs for liquidity provision to summarize how the payoffs vary with the iceberg states. Table 8 reports the average values for the marginal payoffs to liquidity provision by order book level and iceberg state and reports the number of significant negative and positive estimates. The top row reports the average values for $\delta_{1}^{v i s}$ and the top row of the second

half of the table reports the corresponding values for $\delta_{2}^{v i s}$. Twenty-six of the stocks have negative $\delta_{1}^{v i s}$ estimates $(8+5+13)$ and positive $\delta_{2}^{v i s}$ estimates $(8+7+11)$. This pattern in marginal payoffs is in line with the results of Frey and Grammig (2006) and Sandås (2001).

The second, third, and fourth rows of both panels report the average estimated marginal payoffs as a function of whether the iceberg order is on the same side as the limit (own), the opposite side (opp), and both side (own+opp). The marginal payoffs to liquidity provision tend to be positive when liquidity is provided on the same side as an iceberg order and negative when it is provided on the opposite side. These regularities apply in particular to the best level. For twenty-four of 
the stocks the net $\delta$ is positive in the 'own' case and none are significantly negative compared to twenty-six negative estimates for the baseline case. Similarly, when the iceberg is on the opposite side the estimated net payoff becomes significantly negative for all but one stock. For the second best level the 'own' side effect is comparable whereas the 'opp' effect is indistinguishable from zero in most cases. The marginal payoffs for the cases with an iceberg order on both sides are often insignificantly different from zero which is consistent with the above effects partly offsetting each other.

The positive marginal compensation for liquidity provision at the best level when iceberg orders are present on the same side suggests that traders are not bidding aggressively enough or are bidding relatively less aggressively when it is likely that their limit orders compete with hidden liquidity. Similarly, at the second best quotes the results provide some support for the idea that after controlling for other factors traders bid less aggressively when they are likely to compete with hidden liquidity. A difference between the two levels, which is not reflected here, is that at the best level the marginal order has priority relative to the iceberg order whereas the marginal order at the second best level does not. This effect will be accounted for in the expected surplus calculations in the next subsection.

Conversely, when iceberg orders are on the opposite side we observe that traders are bidding too aggressively. Of course, one may also interpret this as evidence that when iceberg orders are present on the opposite side then liquidity traders tend to determine the marginal prices. Our evidence on the order flow suggests that when iceberg orders are present there is more frequent and larger market orders making trades more likely on both sides of the book although the effect is stronger on the side with the hidden liquidity.

\subsection{Liquidity Provider Surplus}

We compute different surplus estimates for liquidity providers based on the results in section 4.4. Additional details on the surplus calculations are provided in Appendix A2. Table 9 reports the average estimates of the expected liquidity provider surplus. Panel A reports the average expected surplus for all states with (i) no iceberg orders and (ii) states with at least some iceberg orders at 
the best quotes. Average standard errors reported in parentheses account for sampling error in the second-stage and the first-stage estimation error for the model parameters. The standard errors are computed under the assumption that the first- and second-stage errors are independent. The average estimates of the liquidity provider surpluses are positive for both no iceberg and iceberg states but the only the estimates for the first two groups are, on average, significantly different from zero.

For individual stocks the estimates are positive for 28 stocks for the no iceberg states and for 23 stocks for the iceberg states. An expected surplus of 0.7 for large stocks and order books with at least one iceberg order implies that collectively the liquidity providers expect to earn approximately 0.7 basis point per trade after subtracting the price impact. The corresponding bid-ask spread (Table 4) is 4.2 basis points so the expected surplus is approximately a third of the half-spread.

In Panel B the expected liquidity surplus is split by display condition — visible versus hiddenand by iceberg state. The first three rows report the surplus for all visible liquidity for order books (states) with no iceberg orders and order books with at least one iceberg order at the best bid or ask quote 8 The last row reports the average expected surplus for the hidden liquidity (in iceberg states). The estimates are positive for both no iceberg and iceberg states for visible liquidity albeit only the estimates for the large and medium category are significantly different from zero. In general, the estimates are close enough to each other that no meaningful ranking can be established between the three different states. The estimated surplus for hidden liquidity is also positive and often significantly different from zero. The hidden liquidity obtains approximately a third of the liquidity provider surplus.

In Panel $\mathrm{C}$ the liquidity provider surplus is split by the order book level - best quote versus the 2nd and 3rd best quotes - and by state. The top half of the panel reports surplus estimates for the best quote level. The first of the four rows provides the overall estimates and the next row the estimate for states with no iceberg orders, followed by states with iceberg orders on the same side, and iceberg orders on the opposite side of the order book. The bottom half of the panel provides

\footnotetext{
${ }^{8}$ The estimates for the no iceberg case differ slightly from the estimate on the first row of the table because the definition of the states is based only on whether or not there are iceberg orders at the best quote levels. Hence, the no iceberg cases include some iceberg orders that are not at the best bid or ask levels.
} 
the corresponding surplus estimates for the second best quote level. A comparison of the first rows of each block within the panel reveals that the positive overall surplus reported in Panels A and B masks a negative surplus for the best quote level and a correspondingly larger positive surplus for the second best level.

The individual estimates for each stock support this with 25 stocks showing significant negative surplus for the best level and significant positive surplus for the second best level. Several of the exceptions to this pattern are among the lower-priced stocks that have positive surplus for both levels.

The difference between marginal compensation and the overall surplus is particularly clear for the second best order book level when comparing the no iceberg and iceberg (own) states. The marginal compensation increases with an iceberg on the same side (the own case) but the expected surplus declines sharply. The difference is that in the iceberg case the depth ahead of any orders on the second best level is much higher so while the marginal compensation is relatively high the surplus is much less because the execution probability is much lower.

\section{Conclusions}

We show that the hidden liquidity changes the behavior of both liquidity providers and liquidity demanders. In general, periods with hidden liquidity in the order books are associated with greater overall liquidity and more trading suggesting that these are periods in which more of the gains from trade are realized. One interpretation of these findings is that market participants view iceberg orders as positive shock to liquidity. An alternative and not necessarily mutually exclusive interpretation is that iceberg orders tend to be submitted in markets that are, in general, more liquid.

A limitation of our approach is that we take as given the arrival and duration of iceberg orders. The alternatives for a trader who submits the iceberg order may include trading off the exchange or splitting up his order into smaller orders that are submitted to the order book over time. Careful modeling of these trade-offs could yield new insights about the economics of hidden liquidity and the trade-offs between transparency and liquidity. Among other things it may alow us to more 
definitively determine which of the above interpretations is closer to the truth. The study by Bessembinder and Venkataraman (2004) of trading at Euronext demonstrates that both iceberg orders and active trading outside the limit order book coexist. 


\section{References}

Abrokwah, K., and G. Sofianos, 2006, "Accessing Displayed and Non-Displayed Liquidity," Journal of Trading, 1, 47-57.

Aitken, M. J., H. Berkman, and D. Mak, 2001, "The Use of Undisclosed Limit Orders on the Australian Stock Exchange," Journal of Banking \& Finance, 25(8), 1589-1603.

Anand, A., and D. G. Weaver, 2004, "Can Order Exposure Be Mandated?," Journal of Financial Markets, 7(4), 405-426.

Baruch, S., 2005, "Who Benefits from an Open Limit Order Book?," Journal of Business, 78(4), $1267-1306$.

Bauwens, L., and P. Giot, 2000, "The Logarithmic ACD Model: An Application to the Bid-Ask Quote Process of Three NYSE Stocks," Annales D'Économie et de Statistique, 60, 117-149.

Bessembinder, H., M. Panayides, and K. Venkataraman, 2008, "Hidden Liquidity: An Analysis of Order Exposure Strategies in Electronic Stock Markets," working paper, University of Utah.

Bessembinder, H., and K. Venkataraman, 2004, "Does an Electronic Stock Exchange Need an Upstairs Market?," Journal of Financial Economics, 73(1), 3-36.

Biais, B., 1993, "Price Formation and Equilibrium Liquidity in Fragmented and Centralized Markets," Journal of Finance, 48, 157-185.

Biais, B., L. Glosten, and C. Spatt, 2005, "Market Microstructure: A Survey of Microfoundations, Empirical Results, and Policy Implications," Journal of Financial Markets, 8, 217-264.

Bloomfield, R., and M. O'Hara, 1999, "Market Transparency: Who Wins and Who Loses?," Review of Financial Studies, 12(1), 5-35.

— , 2000, "Can Transparent Markets Survive?," Journal of Financial Economics, 55, 425-459.

Boehmer, E., G. Saar, and L. Yu, 2005, "Lifting the Veil: An Analysis of Pre-Trade Transparency at the NYSE," Journal of Finance, 60(1), 783-816. 
Bongiovanni, S., M. Borkovec, and R. Sinclair, 2006, "Let's Play Hide-and-Seek: The Location and Size of Undisclosed Limit Order Volume," Journal of Trading, (1), 783-816.

Boni, L., and C. Leach, 2004, "Expanadable Limit Order Markets," Journal of Financial Markets, 7, 145-185.

D'Hondt, C., and R. De Winne, 2007, "Hide-and-Seek in the Market: Placing and Detecting Hidden Orders," Review of Finance, 11, 663-692.

Engle, R. F., and J. R. Russell, 1998, "Autoregressive Conditional Duration: A New Model for Irregularly Spaced Transactions Data," Econometrica, 66, 1127-1162.

Esser, A., and B. Mönch, 2007, "The Navigation of an Iceberg: The Optimal Use of Hidden Orders," Finance Research Letters, 4, 68-81.

Fleming, M. J., and B. Mizrach, 2008, "The Microstructure of a U.S. Treasury ECN: The BrokerTec Platform," working paper, Federal Reserve Bank of New York.

Flood, M. D., R. Huisman, K. G. Koedijk, and R. J. Mahieu, 1999, "Quote Disclosure and Price Discovery in Multiple-Dealer Financial Markets," Review of Financial Studies, 12, 37-59.

Foucault, T., S. Moinas, and E. Theissen, 2007, "Does Anonymity Matter in Electronic Limit Order Markets?," Review of Financial Studies, 20, 1705-1747.

Frey, S., and J. Grammig, 2006, "Liquidity Supply and Adverse Selection in a Pure Limit Order Book Market," Empirical Economics, 30, 1007-1033.

Glosten, L. R., 1994, "Is the Electronic Limit Order Book Inevitable?," Journal of Finance, 49, $1127-1161$.

Harris, L., 1996, "Does a Large Minimum Price Variation Encourage Order Exposure?," working paper, University of Sourthern California.

Hasbrouck, J., and G. Saar, 2004, "Technology and Liquidity Provision: The Blurring of Traditional Definitions," working paper, New York University. 
Hendershott, T., and C. M. Jones, 2005, "Island Goes Dark: Transparency, Fragmentation, and Regulation," Review of Financial Studies, 18, 743-793.

Madhavan, A., 1995, "Consolidation, Fragmentation, and the Disclosure of Trading Information," Review of Financial Studies, 8, 579-603.

— , 1996, "Security Prices and Market Transparency," Journal of Financial Intermediation, $5,255-283$.

— , 2000, "Market Microstructure: A Survey," Journal of Financial Markets, 3, 205-258.

Madhavan, A., D. Porter, and D. Weaver, 2005, "Should Securities Markets Be Transparent?" Journal of Financial Markets, 8, 266-288.

Moinas, S., 2006, "Hidden Limit Orders and Liquidity in Limit Order Markets," working paper, Toulouse Business School.

O’Hara, M., 1995, Market Microstructure Theory. Blackwell Publishers, Cambridge, MA, 1st edn.

Pagano, M., and A. Röell, 1996, "Transparency and Liquidity: A Comparison of Auction and Dealer Markets with Informed Trading," Journal of Finance, 51, 579-611.

Pardo, A., and R. Pascual, 2006, "On the Hidden Side of Liquidity," working paper, University of Valencia.

Parlour, C., and D. Seppi, 2008, "Limit Order Markets: A Survey," Handbook of Financial Intermediation $\&$ Banking, forthcoming (edited by Boot, A.W.A., and Thakor, A.V., Elsevier).

Sandås, P., 2001, "Adverse Selection and Competitive Market Making: Empirical evidence from a limit order market," Review of Financial Studies, 14, 705-734.

Tuttle, L., 2006, "Hidden Orders, Trading Costs and Information," working paper, University of Kansas. 
Table 1: Descriptive Statistics: Iceberg and Limit Orders

\begin{tabular}{|c|c|c|c|c|c|c|c|c|c|}
\hline & \multirow{3}{*}{$\begin{array}{l}\text { Ticker } \\
\text { Symbol }\end{array}$} & \multicolumn{2}{|c|}{ Iceberg Orders as \% of } & \multirow{3}{*}{$\begin{array}{c}\text { Limit } \\
\text { Size } \\
{[1000 \mathrm{shrs}]}\end{array}$} & \multicolumn{3}{|c|}{ Iceberg Order Size } & \multirow{2}{*}{\multicolumn{2}{|c|}{$\begin{array}{l}\text { Distance: Order Price } \\
\text { To Best Quote [b.p.] }\end{array}$}} \\
\hline & & Shares & Shares & & $\overline{\text { Peak Size }}$ & Total Size/ & Executed Shares/ & & \\
\hline & & Submitted & Executed & & [1000 shrs] & Peak Size & Peak Size & Iceberg & Limit \\
\hline \multirow[t]{9}{*}{ Large } & ALV & 5 & 14 & 0.5 & 1.5 & 8.5 & 5.5 & 2.8 & 3.7 \\
\hline & DBK & 7 & 16 & 0.9 & 2.2 & 7.5 & 5.2 & 1.6 & 3.2 \\
\hline & DCX & 8 & 21 & 1.4 & 2.7 & 6.8 & 5.2 & 2.7 & 5.1 \\
\hline & DTE & 7 & 12 & 5.4 & 10.8 & 4.9 & 3.7 & 6.3 & 6.2 \\
\hline & $\mathrm{EOA}$ & 6 & 15 & 1.0 & 2.1 & 7.4 & 4.9 & 1.9 & 3.9 \\
\hline & MUV2 & 8 & 17 & 0.5 & 1.5 & 8.5 & 5.2 & 2.2 & 3.2 \\
\hline & SAP & 6 & 11 & 0.4 & 1.3 & 8.2 & 4.2 & 3.9 & 3.1 \\
\hline & SIE & 7 & 16 & 1.1 & 2.4 & 6.9 & 4.8 & 3.0 & 3.2 \\
\hline & Mean & 7 & 15 & 1.4 & 3.1 & 7.3 & 4.9 & 3.1 & 3.9 \\
\hline \multirow[t]{8}{*}{ Medium } & BAS & 7 & 17 & 0.9 & 2.0 & 8.0 & 5.1 & 2.5 & 4.4 \\
\hline & BAY & 6 & 14 & 1.4 & 2.6 & 7.1 & 4.8 & 4.7 & 4.3 \\
\hline & BMW & 9 & 20 & 0.8 & 2.1 & 7.3 & 4.8 & 2.8 & 3.0 \\
\hline & HVM & 18 & 25 & 1.4 & 4.2 & 6.9 & 4.8 & 5.3 & 5.3 \\
\hline & IFX & 18 & 22 & 3.1 & 7.2 & 5.7 & 4.6 & 8.6 & 8.6 \\
\hline & RWE & 9 & 18 & 0.9 & 2.2 & 7.3 & 4.9 & 3.0 & 5.3 \\
\hline & VOW & 12 & 23 & 0.8 & 2.1 & 7.7 & 5.1 & 2.4 & 2.9 \\
\hline & Mean & 11 & 20 & 1.3 & 3.2 & 7.1 & 4.9 & 4.2 & 4.8 \\
\hline \multirow[t]{16}{*}{ Small } & ADS & 7 & 7 & 0.2 & 1.3 & 8.3 & 3.0 & 2.1 & 0.0 \\
\hline & ALT & 10 & 11 & 0.4 & 1.2 & 9.0 & 4.1 & 3.7 & 1.9 \\
\hline & $\mathrm{CBK}$ & 8 & 19 & 1.4 & 3.8 & 6.9 & 4.8 & 6.1 & 6.5 \\
\hline & CONT & 7 & 12 & 0.5 & 1.9 & 8.1 & 4.3 & 3.3 & 3.1 \\
\hline & DB1 & 16 & 22 & 0.4 & 1.7 & 7.7 & 4.4 & 2.1 & 2.2 \\
\hline & DPW & 14 & 23 & 1.2 & 3.1 & 6.8 & 4.7 & 5.1 & 5.5 \\
\hline & FME & 7 & 10 & 0.2 & 1.2 & 9.3 & 4.1 & 2.0 & 1.8 \\
\hline & HEN3 & 4 & 9 & 0.2 & 1.2 & 9.1 & 4.8 & 0.0 & 1.6 \\
\hline & LHA & 13 & 21 & 1.3 & 3.4 & 7.1 & 5.2 & 6.7 & 6.9 \\
\hline & LIN & 7 & 10 & 0.4 & 1.3 & 8.5 & 4.0 & 2.4 & 2.3 \\
\hline & MAN & 12 & 18 & 0.5 & 1.9 & 7.6 & 4.8 & 3.5 & 3.6 \\
\hline & MEO & 11 & 15 & 0.5 & 1.6 & 8.4 & 4.9 & 3.0 & 2.8 \\
\hline & $\mathrm{SCH}$ & 9 & 14 & 0.5 & 1.6 & 8.3 & 4.9 & 2.5 & 2.6 \\
\hline & TKA & 10 & 13 & 1.3 & 3.3 & 6.6 & 4.0 & 5.9 & 6.2 \\
\hline & TUI & 12 & 15 & 0.8 & 2.2 & 7.4 & 4.2 & 5.3 & 5.4 \\
\hline & Mean & 10 & 15 & 0.7 & 2.0 & 7.9 & 4.4 & 3.6 & 3.5 \\
\hline All & Mean & 9 & 16 & 1.0 & 2.6 & 7.6 & 4.6 & 3.6 & 3.9 \\
\hline
\end{tabular}

Table 1 reports the percent of the total number of shares submitted and total number of shares executed that is accounted for by iceberg orders. For both percentages we exclude all market and marketable limit orders in the totals. The third column reports the average size of limit orders, followed by the average of the iceberg's peak size, ratio of total size to peak size, and ratio of executed shares to peak size for iceberg orders whose first peak size was executed. The last two columns report the median distance between the same-side best quote and the order price of iceberg and limit orders. 
Table 2: Order Execution and Duration

\begin{tabular}{|c|c|c|c|c|c|c|c|c|c|c|}
\hline & \multirow{4}{*}{$\begin{array}{l}\text { Ticker } \\
\text { Symbol }\end{array}$} & \multicolumn{3}{|c|}{ A: Unconditional } & \multicolumn{6}{|c|}{ B: Matched Sample of Icebergs and Limits } \\
\hline & & \multicolumn{2}{|c|}{ Percent with } & \multirow{3}{*}{$\frac{\text { Duration (dur) }}{\text { dur }_{\text {iceberg }}} / \frac{\text { dur }_{\text {limit }}}{\text {. }}$} & \multicolumn{3}{|c|}{ First Peak } & \multicolumn{3}{|c|}{ Second Peak } \\
\hline & & \multicolumn{2}{|c|}{ partial/full execution } & & \multicolumn{2}{|c|}{ Execution [\%] } & \multirow{2}{*}{$\frac{\text { Time-to-Fill (ttf) }}{\mathrm{ttf}_{\text {iceberg }}} / \overline{\text { ttf }_{\text {limit }}}$} & \multicolumn{2}{|c|}{ Execution [\%] } & \multirow{2}{*}{$\overline{\frac{\text { Time-to-Fill (ttf) }}{\text { ttf }_{\text {iceberg }}} / \overline{\text { ttf }_{\text {limit }}}}$} \\
\hline & & Limit & Iceberg & & Limit & Iceberg & & Limit & Iceberg & \\
\hline \multirow[t]{9}{*}{ Large } & ALV & 13 & 49 & 8.5 & 88 & 90 & 1.1 & 84 & 81 & 0.9 \\
\hline & DBK & 14 & 54 & 7.2 & 87 & 92 & 1.2 & 86 & 89 & 1.0 \\
\hline & $\mathrm{DCX}$ & 15 & 54 & 6.8 & 82 & 90 & 1.0 & 85 & 86 & 1.0 \\
\hline & DTE & 22 & 42 & 3.0 & 91 & 95 & 0.9 & 90 & 81 & 0.9 \\
\hline & $\mathrm{EOA}$ & 13 & 51 & 8.0 & 84 & 93 & 1.3 & 87 & 82 & 1.0 \\
\hline & MUV2 & 16 & 51 & 8.9 & 80 & 85 & 0.8 & 85 & 83 & 0.8 \\
\hline & SAP & 13 & 40 & 8.4 & 89 & 86 & 0.8 & 87 & 72 & 0.7 \\
\hline & SIE & 15 & 50 & 6.9 & 88 & 95 & 1.4 & 87 & 85 & 0.9 \\
\hline & Mean & 15 & 49 & 7.2 & 86 & 91 & 1.1 & 86 & 82 & 0.9 \\
\hline \multirow[t]{8}{*}{ Medium } & BAS & 13 & 47 & 8.0 & 81 & 93 & 1.4 & 87 & 81 & 0.6 \\
\hline & BAY & 14 & 48 & 5.9 & 90 & 97 & 1.0 & 87 & 87 & 1.0 \\
\hline & BMW & 13 & 50 & 6.0 & 86 & 92 & 1.5 & 87 & 83 & 0.9 \\
\hline & HVM & 17 & 51 & 5.9 & 90 & 93 & 0.8 & 90 & 80 & 0.8 \\
\hline & IFX & 21 & 44 & 2.6 & 91 & 93 & 0.7 & 88 & 80 & 1.0 \\
\hline & RWE & 14 & 45 & 6.1 & 90 & 97 & 1.1 & 89 & 83 & 1.0 \\
\hline & VOW & 16 & 55 & 6.1 & 86 & 90 & 0.7 & 84 & 83 & 0.6 \\
\hline & Mean & 16 & 49 & 5.8 & 88 & 94 & 1.0 & 87 & 82 & 0.8 \\
\hline \multirow[t]{16}{*}{ Small } & ADS & 12 & 43 & 7.6 & 77 & 80 & 1.6 & 80 & 66 & 0.4 \\
\hline & ALT & 14 & 40 & 5.0 & 87 & 85 & 0.9 & 84 & 75 & 0.4 \\
\hline & CBK & 11 & 54 & 8.1 & 90 & 85 & 1.2 & 87 & 81 & 0.6 \\
\hline & CONT & 12 & 48 & 9.4 & 79 & 92 & 1.4 & 81 & 84 & 0.3 \\
\hline & DB1 & 13 & 46 & 7.6 & 85 & 86 & 0.8 & 86 & 77 & 0.4 \\
\hline & DPW & 16 & 51 & 6.4 & 88 & 96 & 1.3 & 90 & 87 & 0.9 \\
\hline & FME & 10 & 39 & 8.2 & 77 & 82 & 1.4 & 79 & 74 & 0.5 \\
\hline & HEN3 & 9 & 48 & 11.3 & 80 & 94 & 1.1 & 81 & 75 & 0.3 \\
\hline & LHA & 15 & 52 & 7.3 & 87 & 91 & 1.3 & 84 & 81 & 0.7 \\
\hline & LIN & 11 & 37 & 8.7 & 84 & 85 & 0.5 & 86 & 67 & 0.4 \\
\hline & MAN & 13 & 47 & 8.6 & 82 & 88 & 1.0 & 83 & 78 & 0.6 \\
\hline & MEO & 16 & 44 & 7.0 & 90 & 91 & 0.6 & 87 & 79 & 0.5 \\
\hline & $\mathrm{SCH}$ & 15 & 49 & 6.3 & 89 & 90 & 1.0 & 86 & 83 & 0.6 \\
\hline & TKA & 15 & 48 & 6.5 & 88 & 89 & 0.5 & 83 & 67 & 0.8 \\
\hline & TUI & 15 & 46 & 6.3 & 81 & 88 & 1.1 & 85 & 68 & 1.1 \\
\hline & Mean & 13 & 46 & 7.6 & 84 & 88 & 1.0 & 84 & 76 & 0.6 \\
\hline All & Mean & 14 & 47 & 7.1 & 86 & 90 & 1.0 & 86 & 79 & 0.7 \\
\hline
\end{tabular}

Panel A of Table 2 reports the percentage of limit and iceberg orders with at least one execution and the ratio of the median durations of iceberg and limit orders. Order duration is measured from the submission until the time of the last order execution or cancellation. Panel B has two sub-groups; First Peak and Second Peak. First Peak includes all iceberg orders with relative order prices - measured from the best same-side quote-and order sizes close to the median values. A matching limit order sample is constructed by matching on size and relative order price. Second Peak includes iceberg orders whose first peak size was executed. The matching limit order sample includes all limit order submissions that undercut the best same-side quote and that have a size that closely matches the modal iceberg peak size. The execution percentage reported for First Peak is the percent of executed first peaks. The time-to-fill ratio is the ratio of median time-to-fill for iceberg and limit orders. For the Second Peak, the median time-to-fill refers to the time it takes for the second peak of the iceberg order to be executed. 
Table 3: Limit Order Books and Iceberg Orders

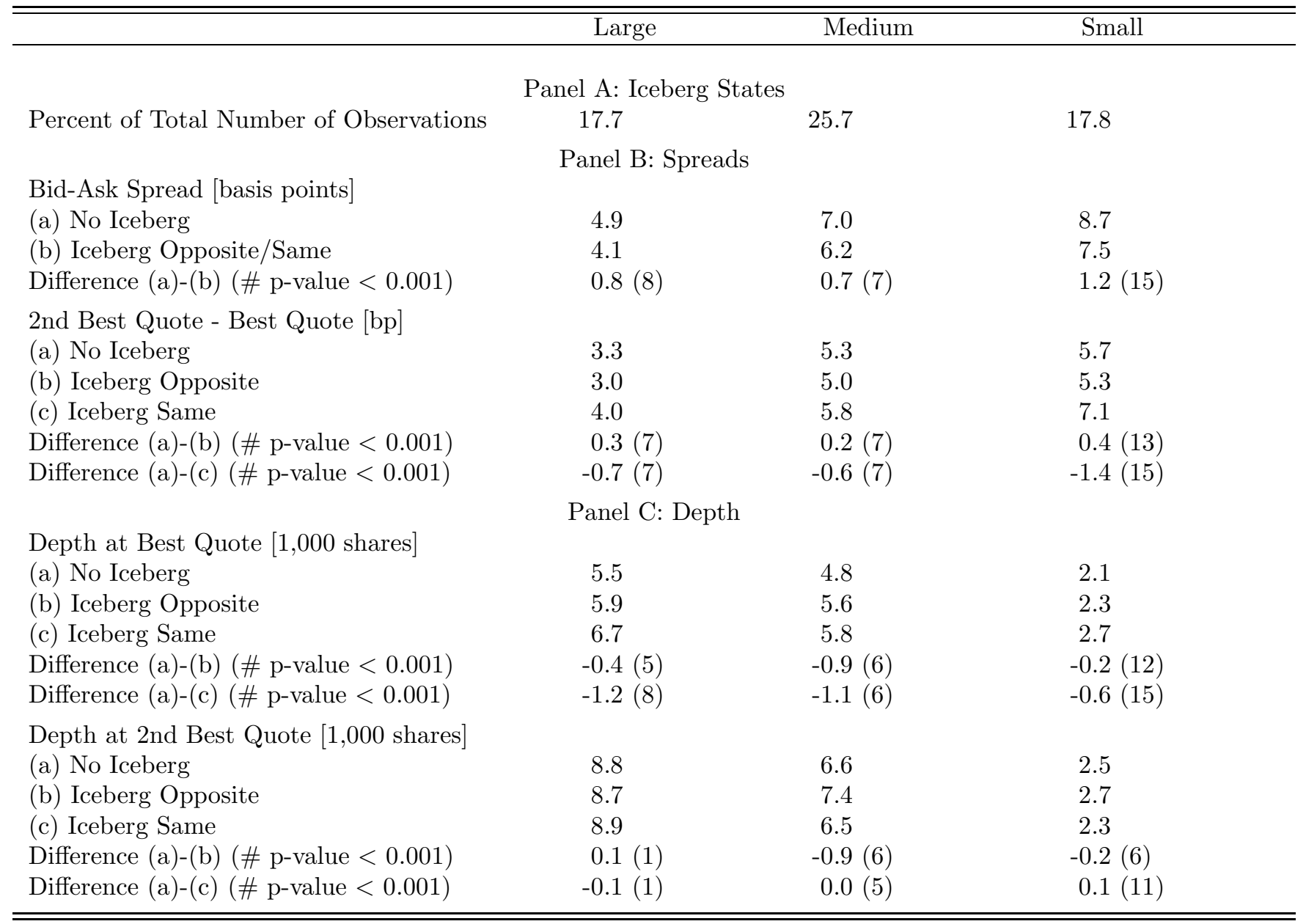

Panel A reports the percent of all observations that have at least one iceberg at the best bid or ask quotes and are classified as iceberg states. Panels B and C report average spreads and depths in the order books observed before transactions according to the iceberg status. The No Iceberg group includes all order books with no iceberg orders at either best quotes. The Iceberg Opposite group includes the bid side of all order books with an iceberg order at the best ask side, and vice versa. The Iceberg Same group includes the bid side of all order books with an iceberg order at the best bid side, and vice versa. All averages are first computed by stock and then averaged across stocks within the large, medium, and small sub-samples. Next to the mean differences, in parenthesis, is the number of stocks within each group that have a mean difference that has the same sign as the overall mean difference and a p-value of 0.001 or less for a test of the null that the difference is zero. 
Table 4: Price Impact and Iceberg Orders

\begin{tabular}{|c|c|c|c|c|c|c|}
\hline & \multicolumn{2}{|c|}{ Large } & \multicolumn{2}{|c|}{ Medium } & \multicolumn{2}{|c|}{ Small } \\
\hline \multicolumn{7}{|c|}{$\begin{array}{c}\text { Panel A: Price Impact Regression: } \\
\Delta m q_{+\tau}=c+\left(a_{0}+a_{1} m\right) d+\left(a_{0}^{h}+a_{1}^{h} m\right) d I^{h}+\left(a_{0}^{n h}+a_{1}^{n h} m\right) d I^{n h}+\epsilon_{+\tau} .\end{array}$} \\
\hline \multicolumn{7}{|c|}{ Time horizon: $\tau=10$ minutes } \\
\hline Intercept & -0.12 & $(0.05)$ & -0.57 & $(0.08)$ & 0.10 & $(0.10)$ \\
\hline$d$ & 1.73 & $(0.07)$ & 2.67 & $(0.10)$ & 3.20 & $(0.14)$ \\
\hline$m d$ & 0.41 & $(0.04)$ & 0.57 & $(0.06)$ & 0.97 & (0.08) \\
\hline$d I^{h}$ & -1.73 & $(0.20)$ & -2.43 & $(0.24)$ & -3.71 & $(0.42)$ \\
\hline$m d I^{h}$ & -0.09 & $(0.09)$ & -0.13 & $(0.11)$ & -0.43 & $(0.18)$ \\
\hline$d I^{n h}$ & 1.94 & $(0.26)$ & 2.61 & $(0.29)$ & 3.86 & $(0.53)$ \\
\hline$m d I^{n h}$ & -0.10 & $(0.12)$ & -0.12 & $(0.14)$ & -0.21 & $(0.25)$ \\
\hline \multicolumn{7}{|c|}{ Time horizon: $\tau=30$ trades } \\
\hline Intercept & -0.14 & $(0.03)$ & -0.28 & $(0.05)$ & -0.19 & $(0.10)$ \\
\hline$d$ & 1.88 & $(0.03)$ & 2.64 & $(0.06)$ & 3.21 & (0.13) \\
\hline$m d$ & 0.46 & $(0.02)$ & 0.63 & $(0.04)$ & 0.99 & (0.08) \\
\hline$d I^{h}$ & -1.84 & $(0.10)$ & -2.26 & $(0.15)$ & -4.14 & $(0.40)$ \\
\hline$m d I^{h}$ & -0.19 & $(0.05)$ & -0.23 & $(0.07)$ & -0.39 & $(0.17)$ \\
\hline$d I^{n h}$ & 1.53 & $(0.13)$ & 2.21 & $(0.18)$ & 4.30 & $(0.51)$ \\
\hline$m d I^{n h}$ & -0.10 & $(0.06)$ & -0.12 & $(0.08)$ & -0.32 & $(0.24)$ \\
\hline \multicolumn{7}{|c|}{ Time horizon: $\tau=$ next trade } \\
\hline Intercept & -0.00 & $(0.00)$ & -0.02 & $(0.01)$ & 0.01 & $(0.02)$ \\
\hline$d$ & 1.00 & $(0.01)$ & 1.61 & $(0.01)$ & 2.02 & $(0.02)$ \\
\hline$m d$ & 0.29 & $(0.00)$ & 0.44 & $(0.01)$ & 0.59 & $(0.01)$ \\
\hline$d I^{h}$ & -1.02 & $(0.02)$ & -1.62 & $(0.03)$ & -2.03 & $(0.07)$ \\
\hline$m d I^{h}$ & -0.18 & $(0.01)$ & -0.23 & $(0.01)$ & -0.35 & $(0.03)$ \\
\hline$d I^{n h}$ & -0.08 & $(0.02)$ & -0.03 & $(0.03)$ & -0.16 & $(0.09)$ \\
\hline$m d I^{n h}$ & -0.02 & $(0.01)$ & -0.06 & $(0.02)$ & -0.07 & $(0.04)$ \\
\hline
\end{tabular}

Panel B: Asymmetric Price Impact Regression:

$\Delta m q_{+\tau}=[$ baseline model as above $] \ldots$

\begin{tabular}{lccc}
\multicolumn{4}{c}{$+\left(\hat{a}_{0}^{h} I^{h}+\hat{a}_{0}^{n h} I^{n h}+\hat{a}_{1} m d+\hat{a}_{1}^{h} m d I^{h}+\hat{a}_{1}^{n h} m d I^{n h}\right) 1_{d=-1}+e_{+\tau}$} \\
\hline \# Rejections: F-Test of $H_{0}: \hat{a}_{0}^{h}=\hat{a}_{0}^{n h}=0$ & 3 & 6 \\
$\tau=10$ minutes & 6 & 1 & 4 \\
$\tau=30$ trades & 2 & 2 & 2 \\
$\tau=$ next trade & 2 & & \\
\# Rejections: F-Test of $H_{0}: \hat{a}_{1}=\hat{a}_{1}^{h}=\hat{a}_{1}^{n h}=0$ & 2 & 3 \\
$\tau=10$ minutes & 5 & 1 & 0 \\
$\tau=30$ trades & 1 & 5 & 11 \\
$\tau=$ next trade & 4 &
\end{tabular}

Panel A reports average parameter estimates and standard errors for price impact regressions with three different time horizons, 10 minutes, 30 trades and next trade. The mid-quote change over the horizon is regressed on a constant $(c)$, and on the trade direction indicator (buy market: $d=1+$, sell market: $d=-1$ ) and the signed normalized market order quantity, $d m$, by themselves and with $d$ and $d m$ interacted with iceberg indicators. The indicator $I^{h}$ is one, if the side of the order book hit by the market order has an iceberg order, for example, buy market order when there is an iceberg at the best ask quote. The indicator $I^{n h}$ is one if the side of the order book that is not hit by the market order has an iceberg order. The midquote changes are measured in basis points and the market order sizes are measured in units of the average market order size. Panel B reports the number of rejegctions for a regression that allows for asymmetric price impact for buy versus sell market orders. 
Table 5: Market Order Flow and Iceberg Orders

\begin{tabular}{|c|c|c|c|}
\hline & Large & Medium & Small \\
\hline & \multicolumn{3}{|c|}{$\begin{array}{l}\text { Panel A: Market Order Size (OLS) } \\
\qquad m=c+b_{1} I^{h}+b_{2} I^{n h}+\epsilon\end{array}$} \\
\hline Intercept & $(0.004)$ & $\begin{array}{ll}0.92 & (0.005)\end{array}$ & $(0.006)$ \\
\hline$I^{h}$ & $(0.010)$ & $0.37 \quad(0.011)$ & $0.50 \quad(0.020)$ \\
\hline \multirow[t]{2}{*}{$I^{n h}$} & $(0.014)$ & $0.18 \quad(0.014)$ & $0.21 \quad(0.026)$ \\
\hline & \multicolumn{3}{|c|}{$\begin{array}{l}\text { Panel B: Probability of a Buy Market Order (Logit) } \\
\qquad \operatorname{Prob}\left(\text { market buy } \mid I^{h}, I^{n h}\right)=\frac{e^{c+b_{1} I^{h}+b_{2} I^{n h}}}{1+e^{c+b_{1} I^{h}+b_{2} I^{n h}}}\end{array}$} \\
\hline$I^{h}$ & $(0.004)$ & $(0.004)$ & $0.14 \quad(0.007)$ \\
\hline \multirow[t]{2}{*}{$I^{n h}$} & $(0.004)$ & $-0.13 \quad(0.004)$ & $-0.14 \quad(0.007)$ \\
\hline & \multicolumn{3}{|c|}{$\begin{array}{c}\text { Panel C: Market Order Duration (log-ACD): } \\
\log \left(E\left[x_{t}\right]\right)=\Psi_{t}=\omega+\alpha \log \left(x_{t-1}\right)+\beta \Psi_{t-1}+b_{1} I^{h}+b_{2} I^{n h}\end{array}$} \\
\hline$\omega$ & $(0.001)$ & $(0.001)$ & $(0.002)$ \\
\hline$\alpha$ & $(0.001)$ & $(0.001)$ & $(0.002)$ \\
\hline$\beta$ & $(0.003)$ & $(0.003)$ & $(0.005)$ \\
\hline$I^{h}$ & $(0.003)$ & $(0.003)$ & $(0.005)$ \\
\hline$I^{n h}$ & $(0.004)$ & $(0.003)$ & $(0.005)$ \\
\hline
\end{tabular}

Panel A reports average parameter estimates with standard errors for the normalized market order size regressed on a constant and iceberg indicators. Panel B reports the cross-sectional means of the marginal effects for a logit model of the probability of a buy market order as a function of the iceberg indicators. The marginal effects are computed for a value of one for the indicator versus a value of zero, and averaged across stocks within each category. The standard errors reported in parenthesis are computed for the average marginal effects for each stock, and are then averaged across stocks within each group. Panel C reports the average parameter estimates for a log-ACD model (see, Bauwens and Giot (2000) and Engle and Russell (1998)) of the (logarithm) of the expected duration between market orders. We remove time-of-day effects by fitting a spline function on thirty minute intervals. The indicator $I^{h}$ is one, if the side of the order book hit by the market order has an iceberg order, for example, buy market order when there is an iceberg at the best ask quote. The indicator $I^{n h}$ is one if the side of the order book that is not hit by the market order has an iceberg order. 
Table 6: Model Summary

$$
\begin{aligned}
& \text { Orderbook Conditions - Ask Side } \\
& E\left[\left[\begin{array}{l}
p^{a s k, 1}-x_{+\tau}\left(q^{a s k, 1}, d=1 ; h^{a s k}, \theta\right)-\delta_{1} h^{a s k} \\
p^{a s k, 2}-x_{+\tau}\left(Q^{a s k, 2}, d=1 ; h^{a s k}, \theta\right)-\delta_{2} h^{a s k}
\end{array}\right] \otimes h^{a s k}\right]=0 \\
& \text { Orderbook Conditions - Bid Side } \\
& E\left[\left[\begin{array}{c}
x_{+\tau}\left(q^{b i d, 1}, d=-1 ; h^{b i d}, \theta\right)-p^{b i d, 1}-\delta_{1} h^{b i d} \\
x_{+\tau}\left(Q^{b i d, 2}, d=-1 ; h^{b i d}, \theta\right)-p^{b i d, 2}-\delta_{2} h^{b i d}
\end{array}\right] \otimes h^{b i d}\right]=0 \\
& \text { Single Tail Expectations - 1st Quote } \\
& x_{+\tau}\left(q^{1}, d ; h, \theta\right)=X+\mu+\left[\alpha_{0} h+\left(\alpha_{1} h\right)\left(q^{1}+\lambda h\right)\right] d \\
& \text { Single Tail Expectations - 2ND Quote } \\
& x_{+\tau}\left(Q^{2}, d ; h, \theta\right)=X+\mu+\left[\alpha_{0} h+\left(\alpha_{1} h\right)\left(Q^{2}+\lambda h+I^{\text {own }} \eta\right)\right] d \\
& E\left[\left[\begin{array}{l}
m-\lambda h^{m k t} \\
X_{+\tau}-X-\mu-\left(\alpha_{0} h^{m k t}\right) d-\left(\alpha_{1} h^{m k t}\right)(d \cdot m) \\
\left(X_{+\tau}-X-\mu-\left(\alpha_{0} h^{m k t}\right) d-\left(\alpha_{1} h^{m k t}\right)(d \cdot m)\right) d \\
\left(X_{+\tau}-X-\mu-\left(\alpha_{0} h^{m k t}\right) d-\left(\alpha_{1} h^{m k t}\right)(d \cdot m)\right)(d \cdot m)
\end{array}\right] \otimes h_{t}^{m k t}\right]=0 \\
& \text { Hidden Depth Conditions } \\
& E\left[\begin{array}{l}
\left(\hat{q}^{a s k}-\eta\right) 1\left(\hat{q}^{a s k}>0\right) \\
\left(\hat{q}^{b i d}-\eta\right) 1\left(\hat{q}^{b i d}>0\right)
\end{array}\right]=0
\end{aligned}
$$




\section{Model Summary - continued}

\section{Model VARiables}

$p^{, 1} \quad p^{, 2}$

$q^{., 1} \quad Q^{., 2}$

$\hat{q}^{b i d} \hat{q}^{a s k}$

$X \quad X_{+\tau}$

$d$

$m \quad f(m ; h, \lambda)$

$I^{\text {ask }} I^{\text {bid }}$

$I^{h} \quad I^{n h}$

$h^{b i d}=\left[\begin{array}{lll}1 & I^{b i d} & I^{a s k}\end{array}\right]$

$h^{a s k}=\left[\begin{array}{lll}1 & I^{a s k} & I^{b i d}\end{array}\right]$

$h^{m k t}=\left[\begin{array}{lll}1 & I^{h} & I^{n h}\end{array}\right]$ 1st and 2nd best bid and ask quotes

order book depth, 1st and cumulative depth 2nd quotes

hidden depth at 1 st quotes

stock price - current and at $t+\tau$

sign of market orders (buy market: $d=+1$, sell market: $d=-1$ )

market order quantity, probability distribution function for $\mathrm{m}$

iceberg indicators for bid and ask side

iceberg and realized market order indicators

$I^{h}=I^{a s k}$ and $I^{n h}=I^{b i d}$, if $d=+1$

$I^{h}=I^{b i d}$ and $I^{n h}=I^{a s k}$, if $d=-1$

iceberg states - bid side view

iceberg states - ask side view

iceberg states - interacted with realized market order

\section{Model Parameters}

$\alpha_{0}=\left[\begin{array}{ll}\alpha_{0}^{v i s} & \alpha_{0}^{\text {own }} \alpha_{0}^{\text {opp }}\end{array}\right]$ fixed component of the price impact

$\alpha_{1}=\left[\begin{array}{ll}\alpha_{1}^{v i s} & \alpha_{1}^{\text {own }} \\ \alpha_{1}^{o p p}\end{array}\right]$ variable component of the price impact

$\mu$

drift of the share price

$\delta_{1}=\left[\begin{array}{lll}\delta_{1}^{\text {vis }} & \delta_{1}^{\text {own }} & \delta_{1}^{o p p}\end{array}\right]$

marginal compensation for liquidity provision at the 1st quote

$\delta_{2}=\left[\begin{array}{lll}\delta_{2}^{\text {vis }} & \delta_{2}^{\text {own }} & \delta_{2}^{\text {opp }}\end{array}\right]$

marginal compensation for liquidity provision at the 2 nd quote

$\lambda=\left[\begin{array}{lll}\lambda^{\text {vis }} & \lambda^{\text {own }} \lambda^{o p p}\end{array}\right]$

$\eta$

mean market order size

mean hidden depth 
Table 7: Model Parameter Estimates

\begin{tabular}{|c|c|c|c|c|c|c|}
\hline \multicolumn{3}{|c|}{ Large } & \multicolumn{2}{|c|}{ Medium } & \multicolumn{2}{|c|}{ Small } \\
\hline \multicolumn{7}{|c|}{ Panel A: Price Impact Function } \\
\hline$\alpha_{0}^{v i s}$ & 1.89 & $(0.08)$ & 2.70 & $(0.23)$ & 3.34 & $(0.33)$ \\
\hline$\alpha_{0}^{\text {own }}$ & -1.90 & $(0.23)$ & -2.08 & $(0.36)$ & -3.60 & $(0.85)$ \\
\hline$\alpha_{0}^{o p p}$ & 1.22 & $(0.25)$ & 2.04 & $(0.42)$ & 3.44 & $(1.14)$ \\
\hline$\alpha_{1}^{v i s}$ & 0.44 & $(0.03)$ & 0.62 & $(0.06)$ & 0.95 & $(0.15)$ \\
\hline$\alpha_{1}^{\text {own }}$ & -0.22 & $(0.04)$ & -0.34 & $(0.06)$ & -0.56 & $(0.15)$ \\
\hline$\alpha_{1}^{o p p}$ & -0.02 & $(0.07)$ & -0.08 & $(0.09)$ & -0.04 & $(0.28)$ \\
\hline$\mu$ & 0.00 & $(0.00)$ & -0.00 & $(0.01)$ & 0.03 & $(0.03)$ \\
\hline \multicolumn{7}{|c|}{ Panel B: Marginal Compensation for Liquidity Provision } \\
\hline$\delta_{1}^{v i s}$ & -0.80 & $(0.09)$ & -1.13 & $(0.20)$ & -1.57 & $(0.41)$ \\
\hline$\delta_{1}^{o w n}$ & 1.82 & $(0.23)$ & 2.59 & $(0.34)$ & 4.06 & $(0.93)$ \\
\hline$\delta_{1}^{o p p}$ & -1.85 & $(0.27)$ & -2.33 & $(0.38)$ & -4.21 & $(1.13)$ \\
\hline$\delta_{2}^{v i s}$ & 1.24 & $(0.17)$ & 2.41 & $(0.31)$ & 2.25 & $(0.66)$ \\
\hline$\delta_{2}^{o w n}$ & 0.50 & $(0.53)$ & 1.75 & $(0.61)$ & 2.28 & $(1.65)$ \\
\hline$\delta_{2}^{o p p}$ & -2.67 & $(0.50)$ & -2.48 & $(0.55)$ & -4.73 & $(1.46)$ \\
\hline \multicolumn{7}{|c|}{ Panel C: Market Order Size and Hidden Depth } \\
\hline$\lambda^{v i s}$ & 0.95 & $(0.00)$ & 0.92 & $(0.01)$ & 0.94 & $(0.01)$ \\
\hline$\lambda^{\text {own }}$ & 0.39 & $(0.01)$ & 0.37 & $(0.02)$ & 0.51 & $(0.04)$ \\
\hline$\lambda^{o p p}$ & 0.12 & $(0.02)$ & 0.17 & $(0.02)$ & 0.18 & $(0.09)$ \\
\hline$\eta$ & 10.91 & $(0.13)$ & 9.05 & $(0.12)$ & 12.35 & $(0.27)$ \\
\hline
\end{tabular}

Table 7 report the average model parameter estimates. The model parameters are estimated using GMM. Table 6 lists the moment conditions and the model parameters. The second stage estimates are computed using a Newey-West 10-lag weighting matrix. The model is estimated for each stock and the average parameter estimates are reported with the average standard errors in parenthesis. Panel A provides the parameters for the price impact function, the $\alpha \mathrm{s}$, and the drift $(\mu)$. Panel B provides the parameters for the marginal compensation for liquidity provision. Panel $\mathrm{C}$ provides the parameters for the market order flow, the $\lambda_{\mathrm{s}}$, and for the mean hidden depth, $(\eta)$. 
Table 8: Marginal Compensation for Liquidity Provision

\begin{tabular}{lrrrrrrrrrr}
\hline \hline & \multicolumn{3}{c}{ Large } & \multicolumn{3}{c}{ Medium } & \multicolumn{3}{c}{ Small } \\
& Mean & + & - & Mean & + & - & Mean & + & - \\
\hline Best Quote Level & & & & & & & & & \\
$\delta_{1}^{\text {vis }}$ & -0.80 & 0 & 8 & -1.13 & 0 & 5 & -1.57 & 0 & 13 \\
$\delta_{1}^{\text {vis }}+\delta_{1}^{\text {own }}$ & 1.02 & 8 & 0 & 1.46 & 7 & 0 & 2.48 & 9 & 0 \\
$\delta_{1}^{\text {vis }}+\delta_{1}^{\text {opp }}$ & -2.66 & 0 & 8 & -3.46 & 0 & 7 & -5.79 & 0 & 14 \\
$\delta_{1}^{\text {vis }}+\delta_{1}^{\text {own }}+\delta_{1}^{\text {opp }}$ & -0.83 & 0 & 4 & -0.87 & 0 & 4 & -1.73 & 0 & 7 \\
& & & & & & & & & \\
Second Best Quote Level & & & & & & & & & \\
$\delta_{2}^{\text {vis }}$ & 1.24 & 8 & 0 & 2.41 & 7 & 0 & 2.25 & 11 & 0 \\
$\delta_{2}^{\text {vis }}+\delta_{2}^{\text {own }}$ & 1.73 & 6 & 0 & 4.15 & 6 & 0 & 4.53 & 9 & 0 \\
$\delta_{2}^{\text {vis }}+\delta_{2}^{\text {opp }}$ & -1.43 & 0 & 3 & -0.07 & 2 & 1 & -2.48 & 1 & 5 \\
$\delta_{2}^{\text {vis }}+\delta_{2}^{\text {own }}+\delta_{2}^{\text {opp }}$ & -0.94 & 2 & 1 & 1.68 & 2 & 0 & -0.20 & 1 & 0 \\
\hline \hline
\end{tabular}

Table 8 reports the mean values of the net marginal compensation for liquidity provision in different states of the order book; no iceberg orders, iceberg order on the same side as the marginal limit (own), iceberg order on the opposite side (opp), and iceberg orders on both sides (own+opp). The units are basis points. The marginal compensation is measured in basis points. The mean value of the estimated parameter values or sums of parameter values are reported and the number of mean values that are significantly different from zero at the $1 \%$ level are reported separately for positive and negative values in the columns labeled " + " and "-." 
Table 9: Liquidity Provider Surplus

\begin{tabular}{|c|c|c|c|c|c|c|}
\hline & \multicolumn{2}{|c|}{ Large } & \multicolumn{2}{|c|}{ Medium } & \multicolumn{2}{|c|}{ Small } \\
\hline \multicolumn{7}{|c|}{ Panel A: By Order Book State } \\
\hline No Iceberg State & 0.44 & $(0.08)$ & 0.87 & $(0.17)$ & 0.64 & $(0.36)$ \\
\hline Iceberg State & 0.68 & $(0.20)$ & 0.70 & $(0.23)$ & 1.08 & $(0.84)$ \\
\hline \multicolumn{7}{|c|}{ Panel B: By Display Condition } \\
\hline Visible Liquidity & 0.45 & $(0.07)$ & 0.74 & $(0.14)$ & 0.56 & $(0.33)$ \\
\hline No Iceberg State & 0.44 & $(0.08)$ & 0.86 & $(0.17)$ & 0.63 & $(0.36)$ \\
\hline Iceberg State & 0.48 & $(0.16)$ & 0.42 & $(0.18)$ & 0.53 & $(0.67)$ \\
\hline Hidden Liquidity & 0.19 & $(0.06)$ & 0.25 & $(0.07)$ & 0.53 & $(0.24)$ \\
\hline \multicolumn{7}{|c|}{ Panel C: By Order Book Level } \\
\hline Best Level & -0.16 & $(0.05)$ & -0.30 & $(0.09)$ & -0.60 & $(0.22)$ \\
\hline No Iceberg State & -0.28 & $(0.05)$ & -0.41 & $(0.11)$ & -0.76 & $(0.23)$ \\
\hline Iceberg Same & 0.83 & $(0.13)$ & 0.86 & $(0.15)$ & 1.80 & $(0.52)$ \\
\hline Iceberg Opposite & -0.46 & $(0.06)$ & -0.83 & $(0.09)$ & -1.35 & $(0.34)$ \\
\hline Second and Third Best Level & 0.61 & $(0.02)$ & 1.04 & $(0.05)$ & 1.16 & $(0.12)$ \\
\hline No Iceberg State & 0.71 & $(0.03)$ & 1.27 & $(0.06)$ & 1.39 & $(0.14)$ \\
\hline Iceberg Same & 0.07 & $(0.00)$ & 0.16 & $(0.01)$ & 0.13 & $(0.02)$ \\
\hline Iceberg Opposite & 0.04 & $(0.03)$ & 0.27 & $(0.04)$ & -0.02 & $(0.15)$ \\
\hline
\end{tabular}

Table 9 reports the estimated expected surplus accruing to liquidity providers. The expected surplus is computed using the parameter estimates for the state-dependent market order distribution and price impact function and the empirical frequencies of buy versus sell orders in each state. The surplus is calculated by integrating the observed price schedule minus the price impact function with respect to the distribution of market order quantities. Panel A reports the surplus for all states with (i) no iceberg orders and (ii) states with at least one iceberg order at the best quotes. In panel B the expected liquidity surplus is split by displayed condition-displayed liquidity versus non-displayed liquidity - and by state. In panel $\mathrm{C}$ the liquidity provider surplus is split by the order book level-best quote versus the 2nd and 3rd best quotes - and by state. Standard errors in parenthesis take into account first-stage estimation error for the model parameters. 


\section{Appendix}

\section{A1 Detecting Iceberg Orders}

We construct an iceberg detection algorithm that uses the order book dynamics to make predictions of whether or not the bid or the ask side has an iceberg order. The algorithm generates informative signals and demonstrates that detection of iceberg orders is feasible. D'Hondt and De Winne (2007) present a similar algorithm and comparable results. In practice, a detection algorithm like the one that we focus on is only one tool that traders may use to detect hidden liquidity. Bongiovanni, Borkovec, and Sinclair (2006) use other observable information to detect hidden orders. Traders may also use active strategies to detect iceberg orders. For example, Hasbrouck and Saar (2004) show that traders may be using very short-lived (fleeting) limit orders to detect possible hidden liquidity on INET. When dealing with multiple venues an algorithm may also keep track of the venues that are most likely to have additional hidden liquidity (Abrokwah and Sofianos (2006)).

\section{A1.1 An Algorithm}

When a trade occurs that involves the execution of volume in excess of the visible volume at given price, it is very likely that the price contains an iceberg order. The ratio between total size to peak size in Table 1 implies that after the replenishing of one peak, additional hidden depth is to typically expected. This implies that one can detect iceberg orders by comparing the recent history of transaction prices and volume with the transition of the visible order book.

Our algorithm sets an iceberg-indicator to one every time new depth is added at a price consistent with the replenishment of an iceberg order. The algorithm resets the indicator for that price to zero only when an event occurs that could not have occurred had the iceberg order remained at that price. The algorithm stores a specific indicator and the expected volume until the next replenishment for each price level. The indicator remains unchanged unless a predicted replenishment fails to occur. The following example illustrates how the algorithm works.

The algorithm maintains and updates a detection vector with the following four elements for all price levels in the order book: price; detection flag; visible volume; volume until next replenishment. In the example, the best bid is initially 9.70 and there are no iceberg orders at this price level, i.e., the detection flag is zero. The first two elements (price and detection flag) are listed in the right-side margin.

1. A buy iceberg order is submitted at 9.77 with a total size of 9000 , a peak size of 1000 , implying a hidden volume of 8000 and a visible volume of 1,000 shares. The detection flag is currently zero. Detection vector: $[9.77,0,1000,0]$.

2. A sell market order for 1200 shares is submitted. The iceberg order's first peak size of 1000 shares is completely executed and another 200 share are automatically executed from the iceberg order's second peak size of 1000 shares. The remaining 800 shares of the iceberg order's second peak size are displayed in the book. The algorithm sets the detection flag to one and sets the volume until next replenishment to 800 . Detection vector: [9.77, 1, 800, 800].

3. A buy limit order is submitted at 9.79 for 200 shares. The flag for 9.79 is zero. Detection vector: $(9.79,0,200,0)$. There is no change at 9.77 .

4. A sell market order for 500 shares is submitted. 200 shares are executed at 9.79 , and 300 shares are executed against the second peak size of the iceberg order at 9.77. The detection flag at 9.77 remains at one, and the volume until next replenishment is revised to 500. Detection vector: $[9.77,1,500,500]$. 
5. A buy limit order is submitted at 9.77 for 5000 shares. The flag at 9.77 remains at one and volume until replenishment is unchanged because the new limit order is behind the visible 500 share of the iceberg order's second peak size. Detection vector: [9.77, 1, 5500, 500].

6. The iceberg order at 9.77 is cancelled. The detection flag remains at one 9 Detection vector: [9.77, $1,5000,500]$.

7. A sell market order is submitted for 600 shares. The flag is reset to zero as the volume until next replenishment is exceeded without the expected replenishment. Detection vector: $[9.77,0,4500,0]$.

\section{A1.2 Performance}

The algorithm will make both type I and type II errors. We take as the null a prediction of no iceberg orders. Table A2 reports the average frequencies of type I and II errors across the four trading activity groups. The average frequency of type I errors is approximately one percent. The average frequency of type II errors is between three and five percent. Around a third of all iceberg states are not discovered because the iceberg order remains completely unexecuted. Our results are comparable to those of D'Hondt and De Winne (2007).

Of course, we cannot determine to what extent the predictions from our algorithm closely approximates the predictions of the market participants. Conversations with market participants suggest that it is reasonable to assume that active participants are able to collect this type of information. It may also be the case that at least some market participants apply algorithms that generate even more accurate predictions. For example, we have not used information in order sizes or any other characteristics that tends to be different for iceberg orders. We have also explored whether there is any evidence that immediate-or-cancel orders may be used to detect iceberg orders but while such orders are used quite frequently the connection to iceberg states is rather weak.

\section{A1.3 Robustness of Model Parameter Estimates}

We re-estimate the model parameters using the same moment conditions as above, but by letting the indicators generated by the detection algorithm determine the values of the indicator variables. Table A3 provides a comparison of the $\delta$ estimates obtained in the baseline case - labeled "True"and in the case of the detection algorithm labeled "Algorithm." Overall, the parameter estimates are fairly close. The price impact parameters and the order flow parameters are also fairly similar suggestion that our main findings for the compensation for liquidity provision are robust to some degree of uncertainty about the iceberg orders.

\footnotetext{
${ }^{9}$ In principle, the algorithm could make use of the fact that the remaining volume of 5,000 at 9.77 exactly matches the size of the previously submitted limit order to infer that it is very likely that the drop in the visible volume at 9.77 was caused by the iceberg being cancelled. However, if the limit order in question was for 500 shares instead of 5,000 shares, it would be a fifty-fifty chance that the cancellation was due to the iceberg order. Our algorithm has not been optimized with respect to these scenarios so it is possible that its performance could be enhanced.
} 


\section{A2 Surplus Calculation}

The formulas presented in sections 4.4 and ?? are independent of the choice of the market order size distribution. Below we provide details about the surplus calculation given the choice of an exponential distribution.

The expected surplus, $\pi^{k}(q)$, of a liquidity provider for unit $q$ in equation (6) is

$$
\pi^{k}(q)=\operatorname{Pr}(d=1 ; h)\left[p^{k}-X-\mu-\left(\alpha_{0} h\right)-\left(\alpha_{1} h\right)(q+\lambda h)\right]\left[1-e^{-\frac{q}{\lambda h}}\right]
$$

The aggregate expected surplus of a quote, used in equations (77) and (8), in the case of the exponential distribution is calculated by

$$
\begin{aligned}
\Pi=\operatorname{Pr}(d=1 ; h)(\lambda h)\left\{\left[p^{k}-X-\mu-\left(\alpha_{0} h\right)-\left(\alpha_{1} h\right)(\lambda h)\right]\left[e^{-\frac{Q_{s}}{\lambda h}}-e^{-\frac{Q_{e}}{\lambda h}}\right]\right. \\
\left.+\left(\alpha_{1} h\right)\left[Q_{s}\left(e^{-\frac{Q_{s}}{\lambda h}}\right)-Q_{e}\left(e^{-\frac{Q_{e}}{\lambda h}}\right)\right]\right\}
\end{aligned}
$$

where the quote starts at $Q_{s}$ and ends at $Q_{e}$. The two formulas above are valid for the ask side, similar equations can be derived for the bid side.

A two stage approach is applied for the standard errors of the surplus expressions. The first stage considers the uncertainty of the estimated parameters of the GMM model via the delta method. The second stage errors are those of the sample average used to calculate the surplus expressions itself. The two errors are combined assuming independence. Denote $S$ as one of the surplus calculations, then an estimator of the variance of $\mathrm{S} \hat{\sigma}_{S}^{2}$ is derived by the following expression

$$
\hat{\sigma}_{S}^{2}=\frac{\partial S}{\partial \theta^{\prime}} \hat{\mathbf{V}}(\hat{\theta}) \frac{\partial S}{\partial \theta}+s_{S}^{2}
$$

where $\hat{\mathbf{V}}(\theta)$ is the estimated variance matrix of the model parameters and $s_{S}^{2}$ is the sampling variance of the surplus calculation. The gradient vector of the surplus expression to the parameters $\frac{\partial S}{\partial \theta}$ is calculated numerically. 
Table A1: Descriptive Statistics: Sample Stocks

\begin{tabular}{|c|c|c|c|c|c|c|c|c|}
\hline & Ticker & Mkt. Cap & Trad. Vol. & Mid-Quote & Trades & Trade Size & Bid- & Spread \\
\hline & Symbol & {$[\in \mathrm{b}]$} & [eb] & {$[\Theta]$} & [1000] & {$[1000 \mathrm{shrs}]$} & [b.p.] & [ $\in$ cents] \\
\hline \multirow[t]{9}{*}{ Large } & ALV & 33.8 & 18.6 & 99.5 & 289.1 & 0.6 & 4.6 & 4.5 \\
\hline & DBK & 38.2 & 19.8 & 67.6 & 253.4 & 1.2 & 4.0 & 2.7 \\
\hline & DCX & 30.3 & 12.0 & 36.3 & 211.6 & 1.6 & 5.2 & 1.9 \\
\hline & DTE & 34.9 & 22.4 & 15.6 & 284.4 & 5.0 & 7.1 & 1.1 \\
\hline & $\mathrm{EOA}$ & 33.8 & 10.3 & 52.5 & 183.7 & 1.1 & 4.5 & 2.4 \\
\hline & MUV2 & 16.4 & 13.3 & 93.6 & 218.9 & 0.6 & 4.6 & 4.3 \\
\hline & SAP & 27.4 & 11.8 & 131.0 & 179.4 & 0.5 & 4.6 & 6.0 \\
\hline & SIE & 52.9 & 20.6 & 63.7 & 282.5 & 1.1 & 3.9 & 2.5 \\
\hline & Mean & 33.5 & 16.1 & 70.0 & 237.9 & 1.5 & 4.8 & 3.2 \\
\hline \multirow[t]{8}{*}{ Medium } & BAS & 25.4 & 8.0 & 43.1 & 165.1 & 1.1 & 4.7 & 2.0 \\
\hline & BAY & 15.9 & 5.7 & 22.8 & 153.5 & 1.6 & 7.1 & 1.6 \\
\hline & BMW & 12.2 & 5.6 & 34.7 & 134.9 & 1.2 & 5.6 & 1.9 \\
\hline & HVM & 6.6 & 6.3 & 18.5 & 123.7 & 2.8 & 9.2 & 1.7 \\
\hline & IFX & 4.8 & 9.4 & 11.6 & 179.0 & 4.5 & 10.0 & 1.2 \\
\hline & RWE & 12.7 & 6.3 & 33.9 & 148.0 & 1.2 & 5.9 & 2.0 \\
\hline & VOW & 9.7 & 6.7 & 39.2 & 162.8 & 1.0 & 5.2 & 2.0 \\
\hline & Mean & 12.5 & 6.8 & 29.1 & 152.4 & 1.9 & 6.8 & 1.8 \\
\hline \multirow[t]{16}{*}{ Small } & ADS & 4.1 & 2.0 & 92.5 & 62.6 & 0.4 & 6.7 & 6.1 \\
\hline & ALT & 3.3 & 2.0 & 48.8 & 69.9 & 0.6 & 7.4 & 3.6 \\
\hline & CBK & 7.6 & 3.4 & 15.3 & 92.7 & 2.4 & 9.6 & 1.5 \\
\hline & CONT & 4.1 & 1.6 & 31.5 & 64.0 & 0.8 & 8.8 & 2.8 \\
\hline & DB1 & 4.8 & 2.3 & 47.0 & 62.8 & 0.8 & 7.0 & 3.3 \\
\hline & DPW & 6.8 & 2.8 & 18.2 & 84.1 & 1.8 & 9.3 & 1.7 \\
\hline & FME & 1.9 & 0.8 & 53.8 & 39.7 & 0.4 & 9.4 & 5.0 \\
\hline & HEN3 & 3.7 & 1.2 & 65.8 & 44.9 & 0.4 & 7.3 & 4.8 \\
\hline & LHA & 4.5 & 2.8 & 14.1 & 86.5 & 2.3 & 10.6 & 1.5 \\
\hline & LIN & 3.4 & 1.4 & 43.6 & 57.3 & 0.6 & 7.5 & 3.3 \\
\hline & MAN & 2.4 & 1.8 & 27.7 & 67.6 & 0.9 & 9.1 & 2.5 \\
\hline & MEO & 5.0 & 2.5 & 34.8 & 79.0 & 0.9 & 8.4 & 2.9 \\
\hline & $\mathrm{SCH}$ & 7.1 & 3.3 & 41.0 & 97.4 & 0.8 & 6.7 & 2.7 \\
\hline & TKA & 6.4 & 2.4 & 15.8 & 80.7 & 1.9 & 10.7 & 1.7 \\
\hline & TUI & 2.0 & 1.7 & 18.7 & 67.8 & 1.3 & 11.5 & 2.1 \\
\hline & Mean & 4.5 & 2.1 & 37.9 & 70.5 & 1.1 & 8.7 & 3.0 \\
\hline All & Mean & 14.1 & 7.0 & 44.4 & 134.2 & 1.4 & 7.2 & 2.8 \\
\hline
\end{tabular}

The table reports the market capitalization, the trading volume, the average mid-quote, the total number of trades, the average trade size (1000 shares), and the average relative (basis points) and absolute (euro cents) bid-ask spreads for the sample stocks. The market capitalization is calculated using a free-float methodology. It is measured in billions of euros as of December 31st, 2003. 
Table A2: Iceberg Detection Algorithm

\begin{tabular}{|c|c|c|c|c|c|c|c|c|c|}
\hline \multirow[b]{3}{*}{ True State } & \multicolumn{3}{|c|}{ Large } & \multicolumn{3}{|c|}{ Medium } & \multicolumn{3}{|c|}{ Small } \\
\hline & \multicolumn{2}{|c|}{ Prediction } & \multirow[b]{2}{*}{ Sum } & \multicolumn{2}{|c|}{ Prediction } & \multirow[b]{2}{*}{ Sum } & \multicolumn{2}{|c|}{ Prediction } & \multirow[b]{2}{*}{ Sum } \\
\hline & No Iceberg & Iceberg & & No Iceberg & Iceberg & & No Iceberg & Iceberg & \\
\hline No Iceberg & 89.8 & 1.0 & 90.8 & 84.9 & 1.4 & 86.3 & 89.9 & 0.8 & 90.7 \\
\hline Iceberg & 3.0 & 6.2 & 9.2 & 4.5 & 9.1 & 13.7 & 3.1 & 6.2 & 9.3 \\
\hline Sum & 92.8 & 7.2 & 100.0 & 89.4 & 10.6 & 100.0 & 93.0 & 7.0 & 100.0 \\
\hline
\end{tabular}

The table reports the average of the percentages of predictions (no iceberg versus iceberg) generated by the iceberg detection algorithm across the percentages of the true iceberg state (no iceberg versus iceberg). The columns of each $2 \times 2$ matrix correspond to the algorithm's predictions and the rows correspond to the true states. Entries on the diagonal correspond to correct predictions. The average percentage of Type I errors is in the top right corner with Type II errors in the bottom left corner. The results are averaged for the bid and ask side for each stock and averaged across stock for the large, medium, and small sub-samples. 
Table A3: Robustness of Select Parameter Estimates

\begin{tabular}{llllllll}
\hline \hline & & \multicolumn{2}{c}{ Large } & \multicolumn{2}{c}{ Medium } & \multicolumn{2}{c}{ Small } \\
\hline$\delta_{1}$ & true & -0.80 & $(0.09)$ & -1.13 & $(0.20)$ & -1.57 & $(0.41)$ \\
& algorithm & -0.72 & $(0.09)$ & -0.95 & $(0.19)$ & -1.24 & $(0.38)$ \\
$\delta_{1}^{\text {own }}$ & true & 1.82 & $(0.23)$ & 2.59 & $(0.34)$ & 4.06 & $(0.93)$ \\
& algorithm & 1.28 & $(0.22)$ & 1.95 & $(0.34)$ & 3.02 & $(0.87)$ \\
$\delta_{1}^{\text {opp }}$ & true & -1.85 & $(0.27)$ & -2.33 & $(0.38)$ & -4.21 & $(1.13)$ \\
& algorithm & -2.10 & $(0.26)$ & -2.76 & $(0.37)$ & -5.08 & $(1.02)$ \\
$\delta_{2}$ & true & 1.24 & $(0.17)$ & 2.41 & $(0.31)$ & 2.25 & $(0.66)$ \\
& algorithm & 1.22 & $(0.18)$ & 2.64 & $(0.30)$ & 2.65 & $(0.61)$ \\
$\delta_{2}^{\text {own }}$ & true & 0.50 & $(0.53)$ & 1.75 & $(0.61)$ & 2.28 & $(1.65)$ \\
& algorithm & 0.58 & $(0.53)$ & 1.19 & $(0.58)$ & 1.45 & $(1.55)$ \\
$\delta_{2}^{\text {opp }}$ & true & -2.67 & $(0.50)$ & -2.48 & $(0.55)$ & -4.73 & $(1.46)$ \\
& algorithm & -2.70 & $(0.50)$ & -3.22 & $(0.54)$ & -6.08 & $(1.43)$ \\
\hline \hline
\end{tabular}

The table reports the estimates for the $\delta$ parameters of the model for the case in which iceberg states are observed without error - rows labeled 'true' - and the case in which iceberg states are detected using the algorithm — rows labeled 'algorithm.' The average parameter estimates are reported with average standard errors in parentheses. 